\title{
Bruhat Order for Two Flags and a Line
}

\author{
PETER MAGYAR \\ Department of Mathematics, Michigan State University, East Lansing, MI 48824
}

\begin{abstract}
The classical Ehresmann-Bruhat order describes the possible degenerations of a pair of flags in a linear space $V$ under linear transformations of $V$; or equivalently, it describes the closure of an orbit of $\operatorname{GL}(V)$ acting diagonally on the product of two flag varieties.

We consider the degenerations of a triple consisting of two flags and a line, or equivalently the closure of an orbit of GL( $V)$ acting diagonally on the product of two flag varieties and a projective space. We give a simple rank criterion to decide whether one triple can degenerate to another. We also classify the minimal degenerations, which involve not only reflections (i.e., transpositions) in the Weyl group $S_{n}, n=\operatorname{dim}(V)$, but also cycles of arbitrary length. Our proofs use only elementary linear algebra and combinatorics.
\end{abstract}

Keywords: quiver representations, multiple flags, degeneration, geometric order

\section{Introduction}

\subsection{A line and two flags}

We shall deal with certain configurations of linear subspaces in $\mathbb{C}^{n}$ (or any vector space $V$ ). A configuration $F=\left(A, B_{\bullet}, C_{\bullet}\right)$ consists of a line $A \subset \mathbb{C}^{n}$ and two flags of subspaces of fixed dimensions, $B_{\bullet}=\left(B_{1} \subset B_{2} \subset \cdots \subset \mathbb{C}^{n}\right)$ and $C_{\bullet}=\left(C_{1} \subset C_{2} \subset \cdots \subset \mathbb{C}^{n}\right)$. In this Introduction, we restrict ourselves to the case in which $B_{\bullet}, C_{\bullet}$ are full flags: $\operatorname{dim} B_{i}=$ $\operatorname{dim} C_{i}=i$ for $i=0,1,2, \ldots, n$.

Our aim is to describe such configurations up to a linear change of coordinates in $\mathbb{C}^{n}$, and the ways in which more generic configurations can degenerate to more special ones. One could ask this question for configurations of arbitrarily many flags; however in general it is 'wild' problem. The distinguishing feature of our case is that there are only finitely many configuration types $F=\left(A, B_{\bullet}, C_{\bullet}\right)$, as we showed in a previous work [5] with Weyman and Zelevinsky. ${ }^{1}$

For example, there exists a most generic type $F_{\max }$, which degenerates to all other types. It consists of those configurations which can be written in terms of some basis $v_{1}, \ldots, v_{n}$ of $\mathbb{C}^{n}$ as:

$$
A=\left\langle v_{1}+v_{2}+\cdots+v_{n}\right\rangle, \quad B_{i}=\left\langle v_{1}, v_{2}, \ldots, v_{i}\right\rangle, \quad C_{i}=\left\langle v_{n}, v_{n-1}, \ldots, v_{n-i+1}\right\rangle .
$$

(Here $\left\langle v_{1}, v_{2}, \ldots\right\rangle$ means the linear span of $v_{1}, v_{2}, \ldots$ ) There is also a most special configuration type $F_{\min }$ :

$$
A=\left\langle v_{1}\right\rangle, \quad B_{i}=C_{i}=\left\langle v_{1}, v_{2}, \ldots, v_{i}\right\rangle .
$$


The configurations of a more generic type can be made to degenerate to more special ones by letting some of the basis vectors $v_{i}$ approach each other, so that in the limit some of the spaces $A, B_{i}, C_{j}$ increase their intersections. ${ }^{2}$ Geometrically, a configuration type is an orbit of $\mathrm{GL}_{n}(\mathbb{C})$ acting diagonally on the product $\mathbb{P}^{n-1} \times \operatorname{Flag}\left(\mathbb{C}^{n}\right) \times \operatorname{Flag}\left(\mathbb{C}^{n}\right)$, with $F_{\max }$ the open orbit and $F_{\min }$ the unique closed orbit. Degeneration of configuration types means the topological closure of a large orbit contains a smaller orbit.

We seek a simple combinatorial description of all degenerations. The trivial case of $n=2$ is illustrated by a diagram in Section 1.3.

Our problem is directly analogous to the classical case in which the configurations consist of two flags only: $F=\left(B_{\bullet}, C_{\bullet}\right)$. This theory originated with Schubert and Ehresmann; a good introduction is [4]. In this case, the configurations (up to change of basis in $\mathbb{C}^{n}$ ) correspond to permutations $w \in S_{n}$ : the configuration type $F_{w}$ consists of the double flags which can be written as:

$$
B_{i}=\left\langle v_{1}, v_{2}, \ldots, v_{i}\right\rangle, \quad C_{i}=\left\langle v_{w(1)}, v_{w(2)}, \ldots, v_{w(i)}\right\rangle
$$

for some basis $v_{1}, \ldots, v_{n}$ of $\mathbb{C}^{n}$. A configuration type $F_{w}$ is a degeneration of another $F_{y}$ exactly if $w \leq y$ in the Bruhat order on $S_{n}$. Namely, $w \leq y$ iff

$$
\#([i] \cap w[j]) \geq \#([i] \cap y[j])
$$

for all $1 \leq i, j \leq n$, where $[i]:=\{1,2, \ldots, i\}$ and $w[j]:=\{w(1), w(2), \ldots, w(j)\}$. This tableau criterion has the geometric meaning:

$$
\#([i] \cap w[j])=\operatorname{dim}\left(B_{i} \cap C_{j}\right)
$$

for $\left(B_{\bullet}, C_{\bullet}\right)$ of type $F_{w}$. The more special configuration $F_{w}$ has larger intersections among its spaces than the more generic $F_{y}$.

We can also describe the classical Bruhat order in terms of its covers: $w<y$ iff $y=$ $\left(i_{0}, i_{1}\right) \cdot w$ for some transposition $\left(i_{0}, i_{1}\right) \in S_{n}$ and $\ell(y)=1+\ell(w)$, where $\ell(w)$ is the number of inversions of $w$. We can picture this definition in terms of the permutation matrices $M_{w}=\left(m_{i j}\right)$ and $M_{y}=\left(m_{i j}^{\prime}\right)$, where $m_{i j}:=\delta_{w(i), j}, m_{i j}^{\prime}:=\delta_{y(i), j}$. Then $w \lessdot y$ means that we have a pair of entries $m_{i_{0} j_{0}}=m_{i_{1} j_{1}}=1$ with $\left(i_{0}, j_{0}\right)$ northwest of $\left(i_{1}, j_{1}\right)$, and no other 1 's in the rectangle $\left[i_{0}, i_{1}\right] \times\left[j_{0}, j_{1}\right]$; and we flip these two 'diagonal' entries in $M_{w}$ to the corresponding anti-diagonal, obtaining $M_{y}$ :

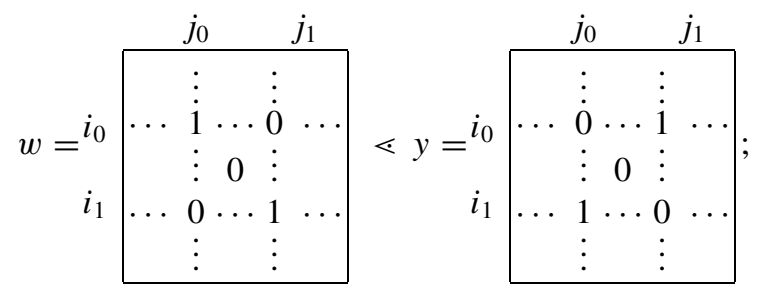

or in compact notation, with 1 replaced by $\bullet$ and all unaffected rows and columns omitted: 


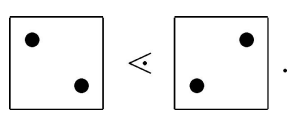

In terms of transpositions: $y=\left(i_{0}, i_{1}\right) \cdot w=w \cdot\left(j_{0}, j_{1}\right)$.

We give a full exposition and proof of these classical results in Sections 2.1 and 3.

\subsection{Bruhat order}

Let us return to our case of a line and two flags. As we showed in [5], we can index our configuration types by decorated permutations $(w, \Delta)$, where $\Delta=\left\{j_{1}<j_{2}<\cdots<j_{t}\right\}$ is any non-empty descending subsequence of $w$, meaning $w\left(j_{1}\right)>w\left(j_{2}\right)>\cdots>w\left(j_{t}\right)$. In the corresponding configuration $F_{w, \Delta}$, the permutation $w$ describes the relative positions of $B_{\bullet}$ and $C_{\bullet}$ in terms of a basis $v_{1}, \ldots, v_{n}$, just as before; and $\Delta$ defines the extra line:

$$
A=\left\langle v_{j_{1}}+v_{j_{2}}+\cdots+v_{j_{t}}\right\rangle .
$$

Thus, the generic $F_{\max }$ is $F_{w, \Delta}$ for $w=w_{0}=n, n-1, \ldots, 2,1$, the longest permutation, and $\Delta=\{1,2, \ldots, n\}$. The most special $F_{\min }$ is $F_{w, \Delta}$ for $w=\mathrm{id}=1,2, \ldots, n$ and $\Delta=\{1\}$. We can picture a decorated permutation as a permutation matrix with circles around the positions $(w(j), j)$ for $j \in \Delta$. For example, $\because \because$ corresponds to $w=\underline{312}$, $\Delta=\{1,3\}$.

We once again have a degeneration or Bruhat order, described combinatorially by a tableau criterion in terms of certain rank numbers which measure intersections of spaces in a configuration $\left(A, B_{\bullet}, C_{\bullet}\right)$ in $F_{w, \Delta}$. Namely, let

$$
r_{i j}(w):=\operatorname{dim}\left(B_{i} \cap C_{j}\right)=\#([i] \cap w[j])
$$

as before, and

$$
\begin{aligned}
r_{\langle i j\rangle}(w, \Delta): & =\operatorname{dim}\left(B_{i} \cap C_{j}\right)+\operatorname{dim}\left(A \cap\left(B_{i}+C_{j}\right)\right) \\
& =\#([i] \cap w[j])+\delta_{i j}(w, \Delta)
\end{aligned}
$$

where

$$
\delta_{i j}(w, \Delta):= \begin{cases}1 & \text { if for all } k \in \Delta, \quad k \leq i \text { or } w(k) \leq j \\ 0 & \text { otherwise. }\end{cases}
$$

We can realize this in terms of linear algebra by defining $\phi_{i j}: B_{i} \times C_{j} \rightarrow \mathbb{C}^{n} / A,\left(v_{1}, v_{2}\right) \mapsto$ $v_{1}+v_{2} \bmod A$ : then $r_{\langle i j\rangle}(w, \Delta)=\operatorname{dim} \operatorname{Ker} \phi_{i j}$. These definitions are suggested by quiver theory: see Section 1.4 below. We will show that our geometric degeneration order has the 
following combinatorial description:

$$
(w, \Delta) \leq(y, \Gamma) \Leftrightarrow\left\{\begin{array}{c}
r_{i j}(w, \Delta) \geq r_{i j}(y, \Gamma) \\
r_{\langle i j\rangle}(w, \Delta) \geq r_{\langle i j\rangle}(y, \Gamma) . \\
\quad \text { for all } 0 \leq i, j \leq n
\end{array}\right.
$$

Finally, we can classify the covers $(w, \Delta) \lessdot(y, \Gamma)$ of our new Bruhat order. Remarkably, in many of the cases below the pair $w<y$ is not a cover in the classical Bruhat order. We describe the covers in terms of certain flipping moves which we write in compact notation (again, with all unaffected rows and columns omitted). We describe how a more generic configuration $\left(A, B_{\bullet}, C_{\bullet}\right)$ (on the right) degenerates to a more special configuration (on the left).

MOVE (i) The line $A$ moves into one of the spaces $B_{i}+C_{j}$, leaving $B_{\bullet}, C_{\bullet}$ unchanged:

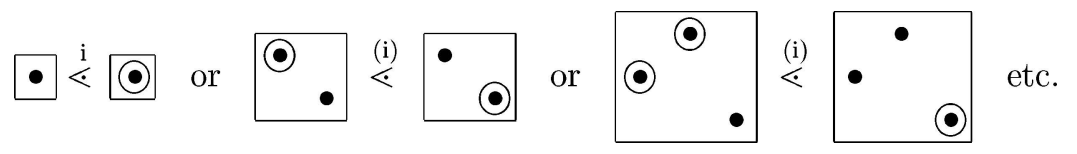

MOVE (ii) One of the $B_{i}$ moves further into one of the $C_{j}$, leaving $A$ unchanged:

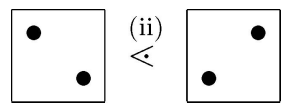

MOVE (iii) The line $A$ lies in $B_{i}+C_{j}$. Then $A$ moves into some $B_{i^{\prime}} \subset B_{i}$, and so does the corresponding line in $C_{j}$. Alternatively, reverse the roles of $B_{i}$ and $C_{j}$.

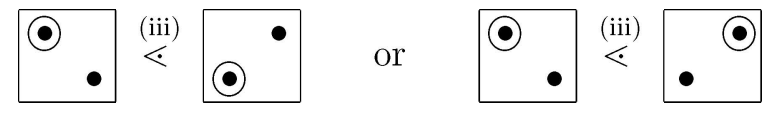

MOVE (iv) The line $A$ lies in $B_{i}-C_{j}$, but not in $B_{i^{\prime}}+C_{j^{\prime}}$, where $B_{i^{\prime}} \subset B_{i}$ and $C_{j^{\prime}} \subset C_{j}$.

Then $A$ moves into $B_{i^{\prime}}+C_{j^{\prime}}$, and the corresponding line in $B_{i}+C_{j}$ moves with it.

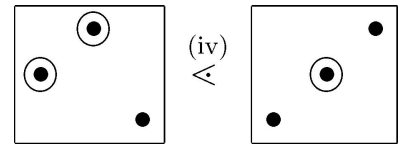


MOVE (v) The line $A$ lies in $B_{i}+C_{j}$. Then $B_{i}$ moves further into $C_{j}$, but $A$ does not move with it, remaining outside $B_{i} \cap C_{j}$.

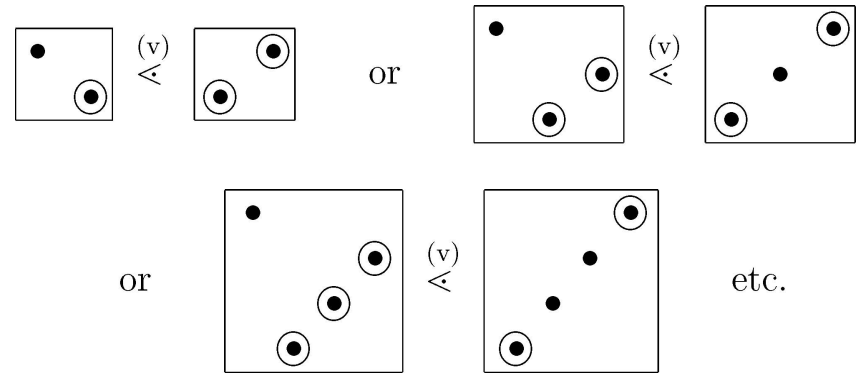

Note that the underlying permutations in this move may differ by an arbitrary-length cycle in $S_{n}$, not necessarily a transposition.

As in the classical case of two flags, certain regions enclosed by the affected dots must be empty for these moves to define covers < (though they always define relations $<$ ). See Section 2.3. The above moves may seem complicated, but they are unavoidable in any computationally effective description: the minimal degenerations are what they are.

We showed in [5] that the number of parameters of a configuration type (i.e., its dimension when thought of as a $\mathrm{GL}_{n}$-orbit in $\mathbb{P}^{n-1} \times \operatorname{Flag}\left(\mathbb{C}^{n}\right) \times \operatorname{Flag}\left(\mathbb{C}^{n}\right)$ ) is:

$$
\operatorname{dim}\left(F_{w, \Delta}\right)=\left(\begin{array}{l}
n \\
2
\end{array}\right)+(n-1)+\ell(w)-\#\left\{\begin{array}{l}
j \mid \begin{array}{c}
\text { for all } k \in \Delta \\
k<j \text { or } w(k)<w(j)
\end{array}
\end{array}\right\} .
$$

For example, $F_{\min }$ has dimension $\left(\begin{array}{c}n \\ 2\end{array}\right)+(n-1)+0-(n-1)=\left(\begin{array}{c}n \\ 2\end{array}\right)$. Indeed, the minimal orbit is isomorphic to $\operatorname{Flag}\left(\mathbb{C}^{n}\right)$.

It is easily seen from the description of the moves (i)-(v), together with the dot-vanishing conditions in Section 2.3, that each move increases the dimension by one. Thus, our Bruhat order is a poset ranked by $\operatorname{dim}(F)-\operatorname{dim}\left(F_{\min }\right)$. (This is no longer true if $\left(B_{\bullet}, C_{\bullet}\right)$ are partial flags, and it is not clear whether our poset is ranked.)

We conjecture that a refinement of the move-labels (i) -(v) on the covers of our poset will give a lexicographic shelling similar to that of Edelman [3] for (undecorated) permutations.

\subsection{Examples $n=2,3$}

We illustrate our constructions in the simplest cases. Let $n=2$. Then the Hasse diagram of our Bruhat order is: 


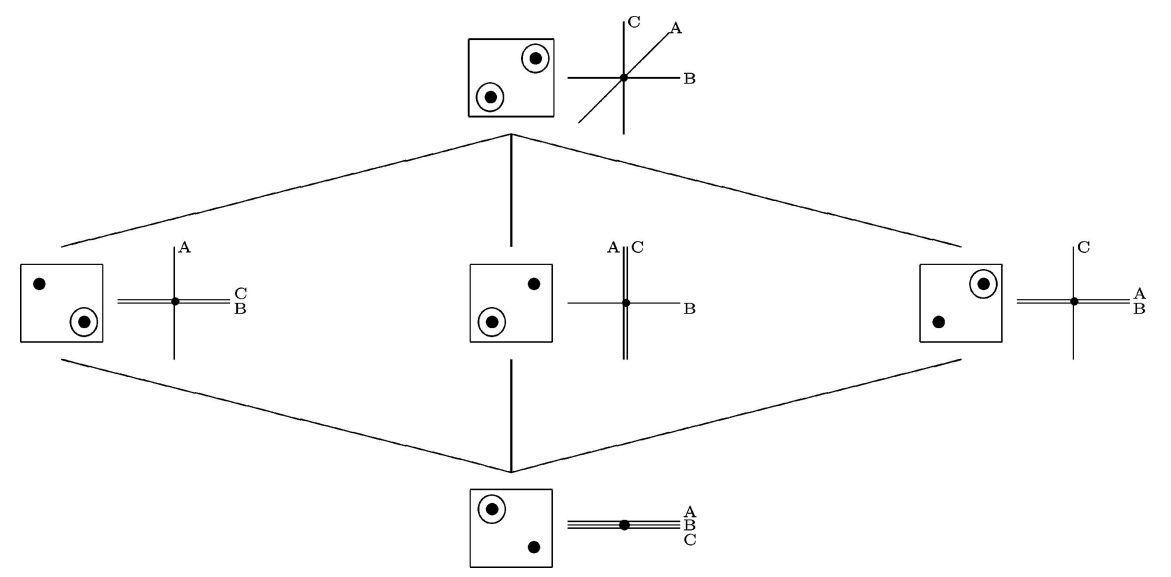

Next to each decorated permutation, we have sketched the corresponding lines $A, B=$ $B_{1}, C=C_{1}$ in $\mathbb{C}^{2}$, with $={ }_{B}^{A}$ indicating that $A$ and $B$ coincide.

The elements of our poset correspond to the $\mathrm{GL}_{2}$-orbits on $\left(\mathbb{P}^{1}\right)^{3}=\left(\mathbb{P}^{1}\right) \times \operatorname{Flag}\left(\mathbb{C}^{2}\right) \times$ Flag $\left(\mathbb{C}^{2}\right)$ : the minimal element is the full diagonal $\mathbb{P}^{1} \subset\left(\mathbb{P}^{1}\right)^{3}$; the mid-level elements are the three partial diagonals, homeomorphic to $\mathbb{P}^{1} \times \mathbb{C}$; and the maximal element is the generic orbit, homeomorphic to $\mathbb{P}^{1} \times \mathbb{C} \times \mathbb{C}^{\times}$, where $\mathbb{C}^{\times}=\mathbb{C} \backslash\{\mathrm{pt}\}$.

Note that this maximal orbit is not a topological cell, even after fibering out $\mathbb{P}^{1}$. For general $n$, the maximal orbit is isomorphic to $\mathrm{GL}_{n} / \mathbb{C}^{\times}=\mathrm{PGL}_{n}$, having fundamental group $\mathbb{Z} / n \mathbb{Z}$. It will be a topic for another paper to understand the geometry of the orbit closures; however this example indicates that they have a non-trivial, but manageable topology.

Now let $n=3$. We can enumerate the configuration types by counting the possible decorations (decreasing subsequences) of each permutation. The identity permutation has $n=3$ decorations, the longest permutation has $2^{n}-1=7$.

$$
\begin{aligned}
& 3+4+4+5+5+7=28 \\
& \underline{123} \underline{213} \underline{132} \underline{231} \underline{312} \underline{321}
\end{aligned}
$$

The Hasse diagram appears on the next page. We have labelled the elements $\min , a, b, \ldots, x$, $y, z$, max, as indicated. For example,

$$
p=(\underline{312},\{1,2\})={ }^{\bullet} \cdot \text {. }
$$

The 72 covering relations, each coming from a move of type (i)-(v), are:

$\begin{array}{ccccccccc}\min \lessdot a & \min \lessdot b & \min \lessdot c & \min \lessdot d & a \lessdot e & a \lessdot f & a \lessdot g & a \lessdot h & \\ b \lessdot f & b \lessdot g & b \lessdot i & b \lessdot j & b \lessdot k & b \lessdot l & c \lessdot h & c \lessdot i & c \lessdot l \\ d \lessdot h & d \lessdot j & d \lessdot k & e \lessdot m & e \lessdot n & f \lessdot n & f \lessdot o & f \lessdot q & \\ g \lessdot n & g \lessdot p & g \lessdot r & h \lessdot m & h \lessdot o & h \lessdot p & h \lessdot q & h \lessdot r & h \lessdot s \\ i \lessdot p & i \lessdot r & i \lessdot s & i \lessdot u & j \lessdot o & j \lessdot t & & & \\ k \lessdot p & k \lessdot q & k \lessdot s & k \lessdot t & l \lessdot p & l \lessdot u & m \lessdot v & m \lessdot w & \\ n \lessdot v & n \lessdot w & n \lessdot y & o \lessdot v & o \lessdot x & o \lessdot y & p \lessdot w & p \lessdot y & p \lessdot z \\ q \lessdot w & q \lessdot x & r \lessdot w & r \lessdot z & s \lessdot x & s \lessdot z & t \lessdot x & t \lessdot y & \\ u \lessdot y & u \lessdot z & v \lessdot \max & w \lessdot \max & x \lessdot \max & y \lessdot \max & & & \end{array}$


The elements in the $i$ th rank of the poset have orbit dimension $i+\operatorname{dim}\left(F_{\min }\right)=i+3$.

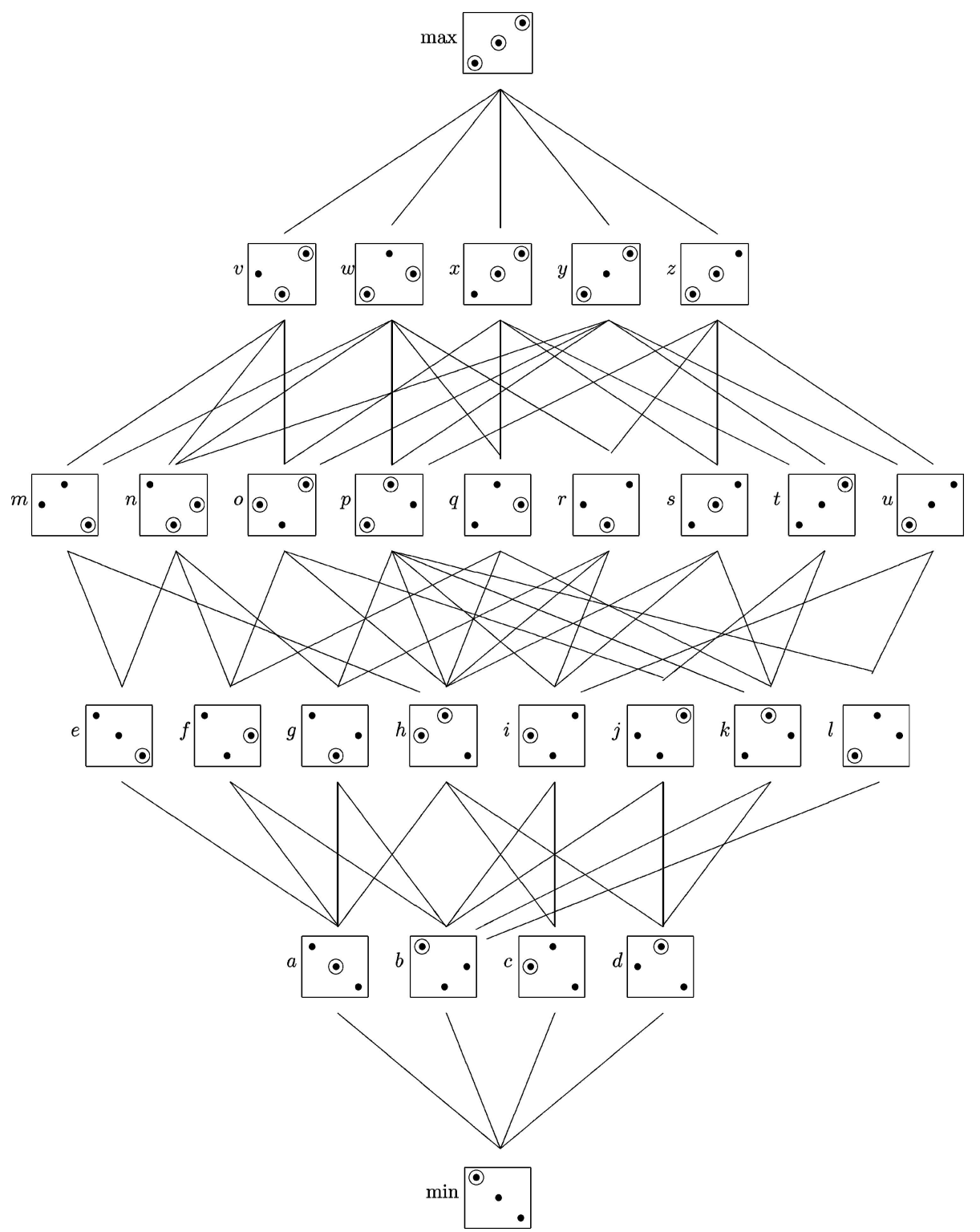

As an illustration of the tableau criterion (i.e., rank numbers defining the Bruhat order), let us check that $e \not z z$ : that is,

$$
\because=(\underline{123},\{3\})=\left(A, B_{\bullet}, C_{\bullet}\right) \quad \text { and } \quad \bullet_{\bullet}^{\bullet}=(\underline{321},\{1,2\})=\left(A^{\prime}, B_{\bullet}^{\prime}, C_{\bullet}^{\prime}\right)
$$


are unrelated elements in our poset, even though $\underline{123}<\underline{321}$ in the classical Bruhat order. Indeed, in the second configuration, $A^{\prime}=\left\langle v_{1}+v_{2}\right\rangle \subset B_{2}^{\prime}=\left\langle v_{1}, v_{2}\right\rangle$, and no degeneration of $\left(A^{\prime}, B_{\bullet}^{\prime}, C_{\bullet}^{\prime}\right)$ can destroy this containment. However, in the first configuration, $A=\left\langle v_{3}\right\rangle \not \subset$ $B_{2}=\left\langle v_{1}, v_{2}\right\rangle$. Thus $(\underline{123},\{3\}) \not \leq(321,\{1,2\})$. In terms of our rank numbers: $r_{i j}(\underline{123}) \geq$ $r_{i j}(\underline{321})$ for all $i, j$, in particular $r_{11}(\underline{123})>r_{11}(\underline{321})$; but $r_{\langle 20\rangle}(\underline{123},\{3\})<r_{\langle 20\rangle}(\underline{321},\{1,2\})$.

\subsection{Structure of the paper}

Now we sketch our proof of the above results. After some easy geometric arguments, we reduce our claims to a rather difficult combinatorial lemma. The idea is to approximate the geometric degeneration order from above and below by combinatorially defined orders, and then show that these combinatorial bounds are equal.

To begin, we distinguish in Section 2 three partial orders on decorated permutations $(w, \Delta)$. First, our geometric order $\stackrel{\operatorname{deg}}{\leq}$ defined by degenerations of the corresponding configuration types $F_{w, \Delta}$. Second, the combinatorial order $\stackrel{\text { rk }}{\leq}$ defined in terms of the rank numbers $r_{i j}(w), r_{v i j\rangle}(w, \Delta)$. Third, the order $\leq$ generated by repeated application of our moves $\lessdot, \ldots, \lessdot$. We wish to show the equivalence of these three orders.

Some simple geometry and linear algebra in Section 4 suffices to show that:

$$
(w, \Delta) \stackrel{\mathrm{mv}}{\leq}(y, \Gamma) \Rightarrow(w, \Delta) \stackrel{\operatorname{deg}}{\leq}(y, \Gamma) \Rightarrow(w, \Delta) \stackrel{\mathrm{rk}}{\leq}(y, \Gamma)
$$

That is, any move corresponds to a degeneration, and any degeneration increases the rank numbers. We are then left in Section 5 to show the purely combinatorial assertion:

$$
(w, \Delta) \stackrel{\text { rk }}{\leq}(y, \Gamma) \Rightarrow(w, \Delta) \stackrel{\text { mv }}{\leq}(y, \Gamma) .
$$

Given a relation $(w, \Delta) \stackrel{\text { rk }}{<}(y, \Gamma)$, we find a move $(w, \Delta) \lessdot(\tilde{w}, \tilde{\Delta})$ such that the smaller rank numbers of $(\tilde{w}, \tilde{\Delta})$ still dominate those of $(y, \Gamma)$ :

$$
(w, \Delta) \stackrel{\mathrm{mv}}{\lessdot}(\tilde{w}, \tilde{\Delta}) \stackrel{\mathrm{rk}}{\leq}(y, \Gamma) .
$$

Iterating this construction within our finite poset, we eventually get

$$
(w, \Delta) \stackrel{\mathrm{mv}}{\lessdot}\left(\tilde{w}_{1}, \tilde{\Delta}_{1}\right) \stackrel{\mathrm{mv}}{\lessdot} \cdots \underset{\leftarrow}{\mathrm{mv}}\left(\tilde{w}_{k}, \tilde{\Delta}_{k}\right)=(y, \Gamma) .
$$

Throughout our proof, we work in the more general case where $B_{\bullet}, C_{\bullet}$ are arbitrary partial flags, with orbits indexed not by permutations but by double cosets of permutations, or "transport matrices", as defined in Section 2.1. Also, our proofs are characteristic-free: our vector spaces are over an arbitrary infinite field $\mathbf{k}$, not necessarily $\mathbb{C}$.

Our Rank Theorem, giving the equivalence of $\stackrel{\text { deg }}{\leq}$ and $\stackrel{\text { rk }}{\leq}$, is a strengthened converse to Proposition 4.5 in our work [5], which relied heavily on quiver theory. In the notation of [5], for a triple flag $X=\left(A, B_{\bullet}, C_{\bullet}\right)$, we have: $r_{i j}(X)=\operatorname{dim} \operatorname{Hom}\left(I_{\{(i, j)\}}, X\right)$ and $r_{\langle i j\rangle}(X)=\operatorname{dim} \operatorname{Hom}\left(I_{\{(i, r),(q, j)\}}, X\right)$. 
Our approach is closely related to that of Zwara and Skowronski [8, 9]; Bongartz [1], Riedtmann [7] et al., who considered the degeneration order on quiver representations. However, in our special case, our results are sharper than those of the general theory. Our description of the covering moves (i)-(v) can be deduced (with non-trivial work) from Zwara's results on the extension order $\underset{\leq}{\operatorname{ext}}$ in [8, 9]. But our results about the rank order $\stackrel{\text { rk }}{\leq}$ are considerably stronger than any of the corresponding general results: our order requires computing Hom with only a few indecomposables, rather than all.

\section{Results}

\subsection{Two flags}

In order to establish the notation for our main theorem in its full generality, we first state the classical theory for two flags.

Throughout this paper, all vector spaces are over a fixed field $\mathbf{k}$ of arbitrary characteristic, infinite but not necessarily algebraically closed; and we fix a vector space $V$ of dimension $n$ with standard basis $e_{1}, \ldots, e_{n}$. Let $\mathbf{b}=\left(b_{1}, \ldots, b_{q}\right)$ be a list of positive integers with sum equal to $n$ : that is, a composition of $n$. We denote by $F \operatorname{lag}(\mathbf{b})$ the variety of partial flags $B_{\bullet}=\left(0=B_{0} \subset B_{1} \subset \cdots \subset B_{q}=V\right)$ of vector subspaces in $V$ such that

$$
\operatorname{dim}\left(B_{i} / B_{i-1}\right)=b_{i} \quad(i=1, \ldots, q)
$$

Flag(b) is a homogeneous space under the natural action of the general linear group $\mathrm{GL}(V)=\mathrm{GL}_{n}(\mathbf{k})$.

Let us fix two compositions of $n, \mathbf{b}=\left(b_{1}, \ldots, b_{q}\right)$ and $\mathbf{c}=\left(c_{1}, \ldots, c_{r}\right)$. The Schubert (or Bruhat) decomposition classifies the orbits of $G L(V)$ acting diagonally on the double flag variety $\operatorname{Flag}(\mathbf{b}) \times \operatorname{Flag}(\mathbf{c})$. We index these orbits by transport matrices $M=\left(m_{i j}\right)$, which are $q \times r$ matrices of nonnegative integers $m_{i j}$ with row sums $b_{i}=\sum_{j} m_{i j}$ and column sums $c_{j}=\sum_{i} m_{i j}$ (so that the sum of all entries is $n$ ). If $\mathbf{b}=\mathbf{c}=(1, \ldots, 1)=\left(1^{n}\right)$, then Flag $(\mathbf{b}) \times$ Flag $(\mathbf{c})$ consists of pairs of full flags, and each transport matrix is the permutation matrix $M=M_{w}$ corresponding to a $w \in S_{n}$, with $m_{w(i), i}=1$ and $m_{i j}=0$ otherwise.

Given a transport matrix $M$, we define the orbit $F_{M} \subset \operatorname{Flag}(\mathbf{b}) \times \operatorname{Flag}(\mathbf{c})$ as the following set of double flags $\left(B_{\bullet}, C_{\bullet}\right)$. Given any basis of $V$ with the $n$ vectors indexed as:

$$
V=\left\langle\begin{array}{l|l}
v_{i j k} & \begin{array}{c}
(i, j) \in[q] \times[r] \\
1 \leq k \leq m_{i j}
\end{array}
\end{array}\right\},
$$

where $[q]=[1, q]:=\{1,2, \ldots, q\}$, let

$$
B_{i}:=\left\langle v_{i^{\prime} j k} \mid i^{\prime} \leq i\right\rangle, \quad C_{j}:=\left\langle v_{i j^{\prime} k} \mid j^{\prime} \leq j\right\rangle,
$$


where \langle\rangle denotes linear span. As the basis $\left\langle v_{i j k}\right\rangle$ varies, $\left(B_{\bullet}, C_{\bullet}\right)$ runs over all double flags in $F_{M}$. In the case $\mathbf{b}=\mathbf{c}=\left(1^{n}\right)$, with $M=M_{w}$, we may take $v_{i j 1}=v_{i}$ for any basis $v_{1}, \ldots, v_{n}$ of $V$, and obtain the configuration type $F_{w}$ from the Introduction.

We can also describe this orbit by intersection conditions:

$$
F_{M}=\left\{\left(B_{\bullet}, C_{\bullet}\right) \mid \operatorname{dim}\left(B_{i} \cap C_{j}\right)=r_{i j}(M)\right\},
$$

where

$$
r_{i j}(M):=\sum_{\substack{(k, l) \\ k \leq i, l \leq j}} m_{k l}
$$

are the rank numbers. This characterization follows from Theorem 1 below.

These orbits cover the double flag variety:

$$
\operatorname{Flag}(\mathbf{b}) \times \operatorname{Flag}(\mathbf{c})=\coprod_{M} F_{M}
$$

where the union runs over all transport matrices $M$.

We shall need the following partial order on the matrix positions $(i, j) \in[1, q] \times[1, r]$ : we write

$$
(i, j) \leq\left(i^{\prime}, j^{\prime}\right) \Leftrightarrow i \leq i^{\prime} \text { and } j \leq j^{\prime}
$$

That is, the northwest positions are small, the southeast positions large. Also, $(i, j)<\left(i^{\prime}, j^{\prime}\right)$ means $(i, j) \leq\left(i^{\prime}, j^{\prime}\right)$ and $(i, j) \neq\left(i^{\prime}, j^{\prime}\right)$, a convention we will use when dealing with any partial order. Furthermore, for sets of positions $\Delta, \Delta^{\prime} \subset[1, q] \times[1, r]$ we let:

$$
\Delta \leq \Delta^{\prime} \Leftrightarrow \forall(i, j) \in \Delta \exists\left(i^{\prime}, j^{\prime}\right) \in \Delta^{\prime} \quad \text { with } \quad(i, j) \leq\left(i^{\prime}, j^{\prime}\right) .
$$

Now, the degeneration order or Ehresmann-Bruhat order on the set of all transport matrices describes how the orbits $F_{M}$ touch each other:

$$
M \stackrel{\text { deg }}{\leq} M^{\prime} \Leftrightarrow \bar{F}_{M} \subset \bar{F}_{M^{\prime}} \Leftrightarrow F_{M} \subset \bar{F}_{M^{\prime}},
$$

where $\bar{F}_{M}$ denotes the (Zariski) closure of $F_{M}$. Our goal is to give a combinatorial characterization of this geometric order.

First, we approximate the degeneration order on double flags by comparing rank numbers. We define:

$$
M \stackrel{\mathrm{rk}}{\leq} M^{\prime} \Leftrightarrow r_{i j}(M) \geq r_{i j}\left(M^{\prime}\right) \quad \forall(i, j) \in[1, q] \times[1, r] .
$$


Second, we define certain moves on matrices which will turn out to be the covers of the degeneration order: that is, the relations $M \stackrel{\text { deg }}{\leftarrow} M^{\prime}$ such that $M \stackrel{\text { deg }}{<} M^{\prime \prime} \stackrel{\text { deg }}{\leq} M^{\prime} \Rightarrow M^{\prime \prime}=M^{\prime}$. Suppose we consider positions $\left(i_{0}, j_{0}\right) \leq\left(i_{1}, j_{1}\right)$ defining a rectangle

$$
R=\left[i_{0}, i_{1}\right] \times\left[j_{0}, j_{1}\right] \subset[1, q] \times[1, r]
$$

and we are given an $M$ satisfying $m_{i_{0} j_{0}}, m_{i_{1} j_{1}}>0$ and $m_{i j}=0$ for all $(i, j) \in R,(i, j) \neq$ $\left(i_{0}, j_{0}\right),\left(i_{1}, j_{1}\right),\left(i_{0}, j_{1}\right),\left(i_{1}, j_{0}\right)$. Then the simple move on the matrix $M$, at the rectangle $R$, is the operation which produces the new matrix:

$$
M^{\prime}=M-E_{i_{0} j_{j}}-E_{i_{1} j_{1}}+E_{i_{0} j_{1}}+E_{i_{1} j_{0}}
$$

where $E_{i j}$ denotes the coordinate matrix with 1 in position $(i, j)$ and 0 elsewhere. We write $M \stackrel{\mathrm{R}}{\leftarrow} M^{\prime}$ or $M \stackrel{\mathrm{mv}}{\leftarrow} M^{\prime}$. Pictorially,

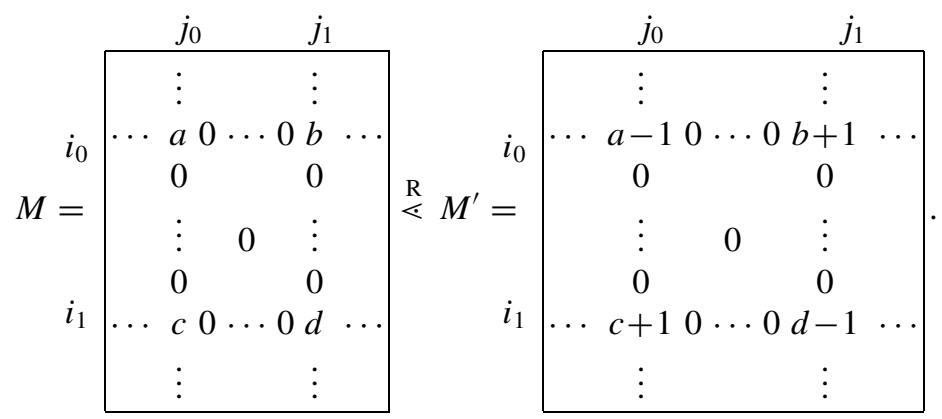

In the case where $M$ is a permutation matrix, the simple move corresponds to multiplying by a transposition: if $M$ is associated to $w$, then $M^{\prime}$ is associated to $w^{\prime}=\left(i_{0}, i_{1}\right) \cdot w=w$. $\left(j_{0}, j_{1}\right)$, and the vanishing conditions on $m_{i j}$ assure that the Bruhat length $\ell\left(w^{\prime}\right)=\ell(w)+1$.

We say $M, M^{\prime \prime}$ are related by the move order, if, starting with $M$, we can perform a sequence of simple moves on various rectangles $R_{1}, R_{2}, \ldots$ to obtain $M^{\prime \prime}$ :

$$
M \stackrel{\mathrm{mv}}{\leq} M^{\prime \prime} \Leftrightarrow M \stackrel{\mathrm{R}_{1}}{\leftarrow} M^{\prime} \stackrel{\mathrm{R}_{2}}{\leftarrow} \cdots M^{\prime \prime} .
$$

Theorem 1 (Ehresmann-Chevalley)

(a) The three orders defined above are equivalent:

$$
M \stackrel{\mathrm{deg}}{\leq} M^{\prime} \Leftrightarrow M \stackrel{\mathrm{rk}}{\leq} M^{\prime} \Leftrightarrow M \stackrel{\mathrm{mv}}{\leq} M^{\prime}
$$

(b) The relation $M \stackrel{\operatorname{deg}}{<} M^{\prime}$ is a cover exactly when $M \stackrel{\text { mv }}{\lessdot} M^{\prime}$.

We give a proof in Section 3. Once we have established the equivalences above, we call the common order the Bruhat order, written simply as $M \leq M^{\prime}$. 


\subsection{A line and two flags}

We now state our main theorems. We consider GL( $V)$ acting diagonally on $\mathbb{P}(V) \times \operatorname{Flag}(\mathbf{b}) \times$ Flag(c), the variety of triples of a line and two flags. We showed in [5] that the orbits correspond to the decorated matrices $(M, \Delta)$, meaning that $M$ is a transport matrix, and

$$
\Delta=\left\{\left(i_{1}, j_{1}\right), \ldots,\left(i_{t}, j_{t}\right)\right\} \subset[1, q] \times[1, r]
$$

is a set of matrix positions satisfying:

$$
i_{1}<i_{2}<\cdots<i_{t}, \quad j_{1}>j_{2}>\cdots>j_{t}, \quad \text { and } \quad m_{i j}>0 \forall(i, j) \in \Delta \text {. }
$$

That is, the positions $\left(i_{1}, j_{1}\right),\left(i_{2}, j_{2}\right), \ldots$ proceed from northeast to southwest. We may concisely write down $(M, \Delta)$ by drawing a circle around the nonzero entries of $M$ at the positions $(i, j) \in \Delta$.

The corresponding orbit $F_{M, \Delta}$ consists of the triple flags $\left(A, B_{\bullet}, C_{\bullet}\right)$ defined as follows. Given a basis $\left\langle v_{i j k}\right\rangle$ as above, the flags $B_{\bullet}, C_{\bullet}$ are defined exactly as before (and thus depend only on $M)$; and the line is defined as $A:=\left\langle\sum_{(i, j) \in \Delta} v_{i j 1}\right\rangle$. Thus $M$ indicates the relative positions of the two flags $B_{\bullet}, C_{\bullet}$, and $\Delta$ is a "decoration" on $M$ indicating the position of the line $A$. Once again we have:

$$
\mathbb{P}(V) \times \operatorname{Flag}(\mathbf{b}) \times \operatorname{Flag}(\mathbf{c})=\coprod_{(M, \Delta)} F_{M, \Delta},
$$

where $(M, \Delta)$ runs over all decorated matrices. We also define the degeneration order $(M, \Delta) \stackrel{\leq}{\leq}\left(M^{\prime}, \Delta^{\prime}\right)$ as before.

Next we define the rank order. For $\left(A, B_{\bullet}, C_{\bullet}\right) \in F_{M, \Delta}$, we define a new rank number:

$$
\begin{aligned}
r_{\langle i j\rangle}(M, \Delta) & :=\operatorname{dim}\left(B_{i} \cap C_{j}\right)+\operatorname{dim}\left(A \cap\left(B_{i}+C_{j}\right)\right) \\
& =r_{i j}(M)+\delta_{i j}(\Delta)
\end{aligned}
$$

where we define:

$$
\begin{aligned}
\delta_{i j}(\Delta) & := \begin{cases}1 & \text { if } \Delta \leq\{(i, r),(q, j)\} \\
0 & \text { otherwise }\end{cases} \\
& = \begin{cases}0 & \text { if }(i+1, j+1) \leq \Delta \\
1 & \text { otherwise }\end{cases}
\end{aligned}
$$

We can extend this definition to $(i, j) \in[0, q] \times[0, r]$ by setting $r_{i j}(M):=0$ if $i=0$ or $j=0$, so that

$$
r_{\langle i 0\rangle}(M, \Delta)=\operatorname{dim}\left(A \cap B_{i}\right)=\delta_{i 0}(\Delta), \quad r_{\langle 0 j\rangle}(M, \Delta)=\operatorname{dim}\left(A \cap C_{j}\right)=\delta_{0 j}(\Delta) .
$$


Now we let:

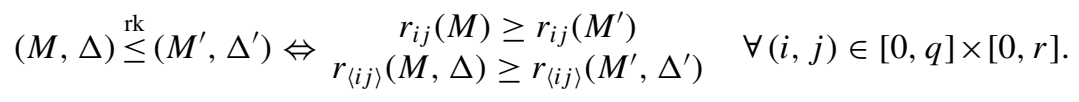

Rank Theorem The degeneration order and the rank order are equivalent:

$$
(M, \Delta) \stackrel{\operatorname{deg}}{\leq}\left(M^{\prime}, \Delta^{\prime}\right) \Leftrightarrow(M, \Delta) \stackrel{\mathrm{rk}}{\leq}\left(M^{\prime}, \Delta^{\prime}\right)
$$

That is, the triple flag $\left(A, B_{\bullet}, C_{\bullet}\right)$ is a degeneration of $\left(A^{\prime}, B_{\bullet}^{\prime}, C_{\bullet}^{\prime}\right)$ if and only if, for all $(i, j) \in[0, q] \times[0, r]$,

$$
\begin{gathered}
\operatorname{dim}\left(B_{i} \cap C_{j}\right) \geq \operatorname{dim}\left(B_{i}^{\prime} \cap C_{j}^{\prime}\right) \\
\operatorname{dim}\left(B_{i} \cap C_{j}\right)+\operatorname{dim}\left(A \cap\left(B_{i}+C_{j}\right)\right) \geq \operatorname{dim}\left(B_{i}^{\prime} \cap C_{j}^{\prime}\right)+\operatorname{dim}\left(A^{\prime} \cap\left(B_{i}^{\prime}+C_{j}^{\prime}\right)\right) .
\end{gathered}
$$

\subsection{Simple moves}

Below, we define simple moves of types (i)-(v) on a decorated matrix $(M, \Delta)$, each producing a new matrix $\left(M^{\prime}, \Delta^{\prime}\right)$, so that we write $(M, \Delta) \stackrel{\text { mv }}{\leftarrow}\left(M^{\prime}, \Delta^{\prime}\right)$. Given these moves, we define the move order $(M, \Delta) \stackrel{\text { mv }}{\leq}\left(M^{\prime \prime}, \Delta^{\prime \prime}\right)$ as before.

Move Theorem The degeneracy order and the move order are equivalent:

$$
(M, \Delta) \stackrel{\operatorname{deg}}{\leq}\left(M^{\prime}, \Delta^{\prime}\right) \Leftrightarrow(M, \Delta) \stackrel{\mathrm{mv}}{\leq}\left(M^{\prime}, \Delta^{\prime}\right)
$$

Again, we call the common order the Bruhat order.

Minimality Theorem The relation $(M, \Delta) \stackrel{\operatorname{deg}}{<}\left(M^{\prime}, \Delta^{\prime}\right)$ is a cover exactly when $(M, \Delta) \stackrel{\mathrm{mv}}{\leftarrow}$ $\left(M^{\prime}, \Delta^{\prime}\right)$ for one of the simple moves (i)-(v).

We introduce an operation which normalizes an arbitrary subset $S$ of matrix positions into a decoration $\Delta$ of the prescribed form. For $S \subset[1, q] \times[1, r]$, let:

$$
[S]:=\{(i, j) \in S \mid(i, j) \nless(k, l) \forall(k, l) \in S\}
$$

be the set of $\leq$-maximal positions in $S$. This operation is "explained" by the following lemma, proved in Section 5:

Lemma 1 (Uncircling lemma) If $M$ is a transport matrix, $S$ a set of matrix positions with $m_{i j}>0$ for all $(i, j) \in S$, and we define $\left(A, B_{\bullet}, C_{\bullet}\right)$ by the same formulas as for a decorated matrix (namely $A:=\left\langle\sum_{(i, j) \in S} v_{i j 1}\right\rangle$ ), then $\left(A, B_{\bullet}, C_{\bullet}\right) \in F_{M, \Delta}$, where $\Delta=[S]$.

It remains to define the five types of simple moves $(M, \Delta) \stackrel{(\mathrm{i})}{\leftarrow}\left(M^{\prime}, \Delta^{\prime}\right), \ldots,(M, \Delta) \stackrel{(\mathrm{v})}{\leftarrow}$ $\left(M^{\prime}, \Delta^{\prime}\right)$. Although geometrically, it is natural to think of the more general configuration degenerating to the more special one, combinatorially it is more convenient to describe 
the modification of the more special (smaller) element $(M, \Delta)$ to obtain the more general (larger) element $\left(M^{\prime}, \Delta^{\prime}\right)$.

In each case, we indicate the matrix positions in $(M, \Delta)$ modified by the move, and we list the requirements on $M=\left(m_{i j}\right)$ and $\Delta$ for the move to be valid. Then we specify the result of the move, $\left(M^{\prime}, \Delta^{\prime}\right)$.

(i) Suppose we have a position $\left(i_{1}, j_{1}\right) \not \leq \Delta$, with $m_{i_{1} j_{1}}>0$ and $m_{i j}=0$ whenever $(i, j)<\left(i_{1}, j_{1}\right),(i, j) \not \leq \Delta$. Then define:

$$
M^{\prime}:=M, \quad \Delta^{\prime}:=\left[\Delta \cup\left\{\left(i_{1}, j_{1}\right)\right\}\right] .
$$

$(M, \Delta)$

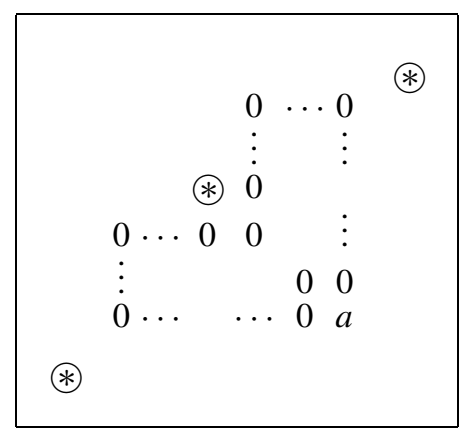

$\left(M^{\prime}, \Delta^{\prime}\right)$

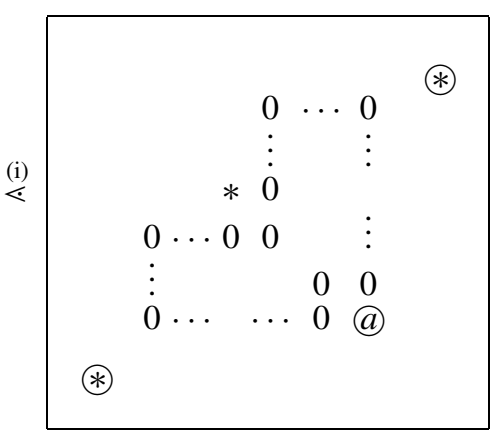

where $a=m_{i_{1} j_{1}}>0$, the symbol $*$ represents a matrix entry $m_{i j}>0$, and a circle $\circledast$ around $*=m_{i j}$ means that $(i, j) \in \Delta$. The values of $m_{i j}$ are not changed by the move.

(ii) Suppose we have positions $\left(i_{0}, j_{0}\right)<\left(i_{1}, j_{1}\right)$ with: $m_{i_{0} j_{0}}, m_{i_{1} j_{1}}>0$, and $m_{i j}=0$ whenever: $\left(i_{0}, j_{0}\right)<(i, j)<\left(i_{1}, j_{1}\right),(i, j) \neq\left(i_{0}, j_{1}\right),\left(i_{1}, j_{0}\right)$. Suppose further that $\left(i_{1}, j_{1}\right) \notin$ $\Delta ;\left(i_{0}, j_{1}\right) \notin \Delta$ or $\left(i_{1}, j_{0}\right) \notin \Delta ;$ and $\left(i_{0}, j_{0}\right) \notin \Delta$ or $m_{i_{0} j_{0}}>1$. Then define:

$$
M^{\prime}:=M-E_{i_{0} j_{0}}-E_{i_{1} j_{1}}+E_{i_{0} j_{1}}+E_{i_{1} j_{0}}, \quad \Delta^{\prime}:=\Delta .
$$

\begin{tabular}{|c|}
\hline$a 0 \cdots 0 b$ \\
\hline $\begin{array}{ll}0 & 0\end{array}$ \\
\hline$\vdots \quad 0$ \\
\hline 0 \\
\hline$c 0 \cdots 0 d$ \\
\hline
\end{tabular}

$(M, \Delta)$
$\left(M^{\prime}, \Delta^{\prime}\right)$

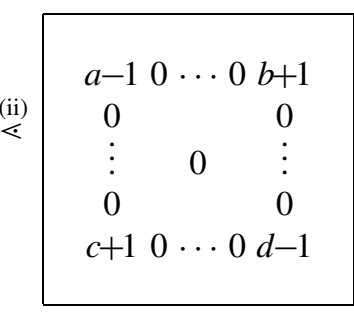

Here $a=m_{i_{0} j_{0}}>0, d=m_{i_{1} j_{1}}>0, a$ may be circled only if $a>1$, at most one of $b, c$ is circled, and $d$ is not circled. The positions of circles are unchanged by the move. 
(iii)(a) Suppose $\left(i_{0}, j_{0}\right)<\left(i_{1}, j_{1}\right)$ with $\left(i_{0}, j_{0}\right) \in \Delta, m_{i_{0} j_{0}}=1, m_{i_{1} j_{1}}>0$, and $m_{i j}=0$ whenever: $\left(i_{0}, j_{0}\right)<(i, j)<\left(i_{1}, j_{1}\right),(i, j) \neq\left(i_{1}, j_{0}\right)$; and whenever $(i, j) \leq\left(i_{0}, j_{1}\right),(i, j) \not \leq$ $\Delta$. Then define:

$$
M^{\prime}:=M-E_{i_{0} j_{0}}-E_{i_{1} j_{1}}+E_{i_{0} j_{1}}+E_{i_{1} j_{0}}, \quad \Delta^{\prime}:=\left[\Delta \cup\left\{\left(i_{0}, j_{1}\right)\right\}\right]
$$

$(M, \Delta)$

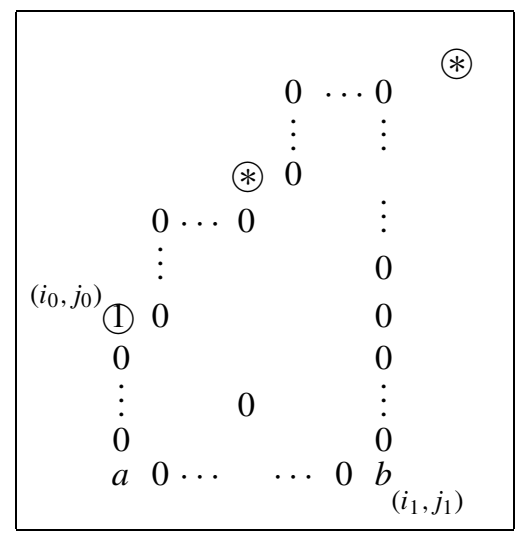

$\left(M^{\prime}, \Delta^{\prime}\right)$

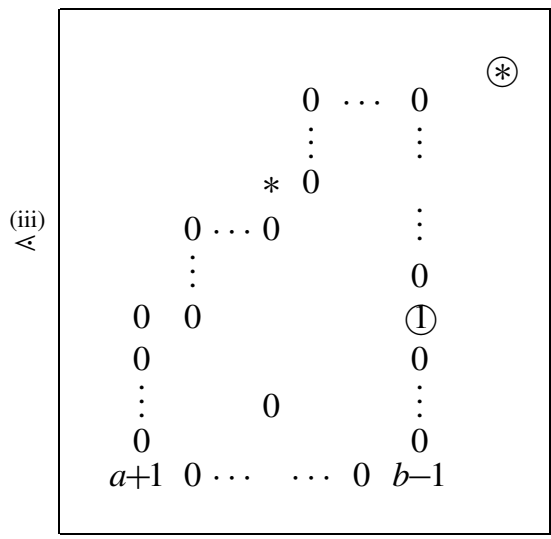

Here the (1) on the left is at position $\left(i_{0}, j_{0}\right), \quad a=m_{i_{1} j_{0}}$, and $b=m_{i_{1} j_{1}}>0$.

(iii)(b) The transpose of move (iii)(a). Suppose $\left(i_{0}, j_{0}\right)<\left(i_{1}, j_{1}\right)$ with $\left(i_{0}, j_{0}\right) \in \Delta, m_{i_{0} j_{0}}=$ $1, m_{i_{1} j_{1}}>0$, and $m_{i j}=0$ whenever: $\left(i_{0}, j_{0}\right)<(i, j)<\left(i_{1}, j_{1}\right),(i, j) \neq\left(i_{0}, j_{1}\right)$; and whenever $(i, j) \leq\left(i_{1}, j_{0}\right),(i, j) \not \leq \Delta$. Then define:

$$
M^{\prime}:=M-E_{i_{0} j_{0}}-E_{i_{1} j_{1}}+E_{i_{0} j_{1}}+E_{i_{1} j_{0}}, \quad \Delta^{\prime}:=\left[\Delta \cup\left\{\left(i_{1}, j_{0}\right)\right\}\right] .
$$

$(M, \Delta)$

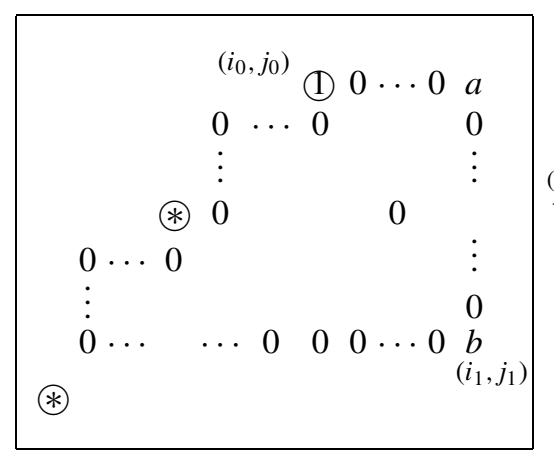

$\left(M^{\prime}, \Delta^{\prime}\right)$

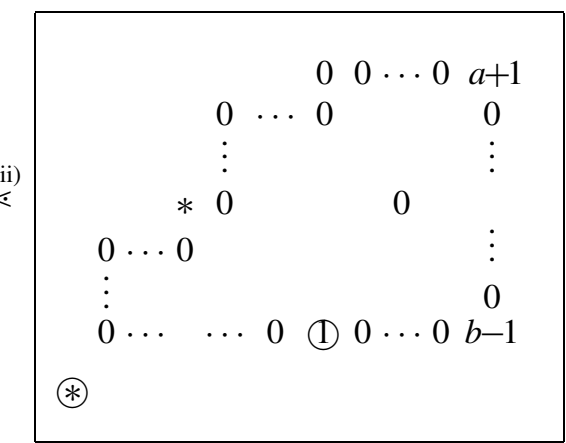

Here the (1) on the left is at position $\left(i_{0}, j_{0}\right), a=m_{i_{0} j_{1}}$, and $b=m_{i_{1} j_{1}}>0$. 
(iv)(a) Suppose $i_{0}<i_{2}<i_{1}$ and $j_{2}<j_{0}<j_{1}$ with $\left(i_{0}, j_{0}\right),\left(i_{2}, j_{2}\right) \in \Delta, m_{i_{0} j_{0}}=m_{i_{2} j_{2}}=1$, $m_{i_{1} j_{1}}>0$, and $m_{i j}=0$ whenever: $\left(i_{0}, j_{2}\right)<(i, j)<\left(i_{1}, j_{1}\right)$ and $(i, j) \not \leq \Delta$ and $(i, j) \neq$ $\left(i_{0}, j_{1}\right),\left(i_{1}, j_{2}\right)$. Then define:

$$
M^{\prime}:=M-E_{i_{0} j_{0}}-E_{i_{1} j_{1}}-E_{i_{2} j_{2}}+E_{i_{1} j_{2}}+E_{i_{2} j_{0}}+E_{i_{0} j_{1}}, \quad \Delta^{\prime}:=\left[\Delta \cup\left\{\left(i_{2}, j_{0}\right)\right\}\right] .
$$

$(M, \Delta)$

$\left(M^{\prime}, \Delta^{\prime}\right)$
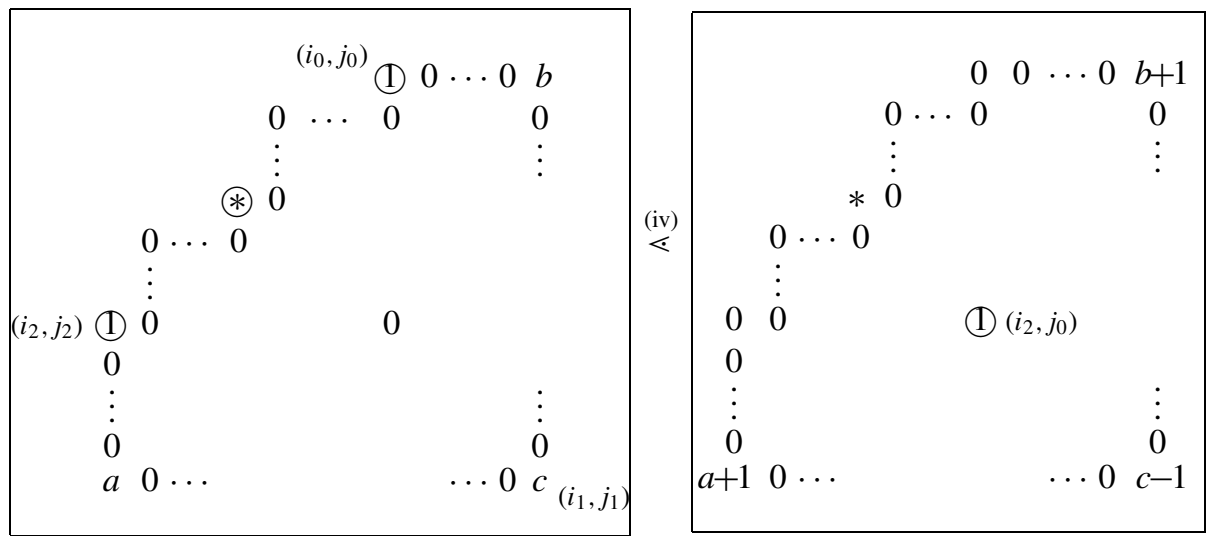

Here the coordinate markings indicate that on the left, the (1)'s are at positions $\left(i_{0}, j_{0}\right)$, $\left(i_{2}, j_{2}\right)$, and $c=m_{i_{1} j_{1}}>0$. The (1) on the right is at position $\left(i_{2}, j_{0}\right)$.

(iv)(bc) Suppose we have $\left(i_{0}, j_{0}\right)<\left(i_{1}, j_{1}\right)$ with $m_{i_{0} j_{0}}, m_{i_{1}, j_{1}}>0$, and $m_{i j}=0$ whenever: $\left(i_{0}, j_{0}\right)<(i, j)<\left(i_{1}, j_{1}\right)$, except for $(i, j)=\left(i_{0}, j_{1}\right),\left(i_{1}, j_{0}\right)$ and one other position as specified below. Further suppose one of the following cases:

(b) we have $i_{0}<i_{2}<i_{1}$ with $\left(i_{2}, j_{0}\right) \in \Delta, m_{i_{2} j_{0}}=1$, and $\left(i_{0}, j_{1}\right) \not \leq \Delta$; or

(c) we have $j_{0}<j_{2}<j_{1}$ with $\left(i_{0}, j_{2}\right) \in \Delta, m_{i_{0} j_{2}}=1$, and $\left(i_{1}, j_{0}\right) \not \leq \Delta$.

Then define:

$$
M^{\prime}:=M-E_{i_{0} j_{0}}-E_{i_{1} j_{1}}+E_{i_{0} j_{1}}+E_{i_{1} j_{0}}, \quad \Delta^{\prime}:=\Delta .
$$

This is the same as move (ii), except that it occurs in the presence of a (1) at $\left(i_{0}, j_{2}\right)$ or $\left(i_{2}, j_{0}\right)$.

(b)

$(M, \Delta)$

\begin{tabular}{|c|c|c|}
\hline $\begin{array}{lllll}a & 0 & \cdots & 0 & b\end{array}$ & & $a-1 \quad 0 \quad \cdots \quad c c b+1$ \\
\hline $\begin{array}{ll}0 & 0\end{array}$ & & $\begin{array}{ll}0 & 0\end{array}$ \\
\hline : & & $:$ \\
\hline $\begin{array}{l}0 \\
\text { (1) }\end{array}$ & $\stackrel{(I v)}{\lessdot}$ & $\stackrel{0}{(1)}$ \\
\hline 0 & & 0 \\
\hline : & & : \\
\hline $0 \quad 0$ & & $\dot{0}$ \\
\hline $\begin{array}{ccccc}c & 0 & \cdots & 0 & d\end{array}$ & & $c+1 \quad 0 \quad \cdots \quad 0 \quad d-1$ \\
\hline
\end{tabular}

$\left(M^{\prime}, \Delta^{\prime}\right)$ 
Here $b$ must not be circled, and there must be no circled element weakly southeast of $b$. The move does not change the circled positions.

(c) $(M, \Delta)$ $\left(M^{\prime}, \Delta^{\prime}\right)$
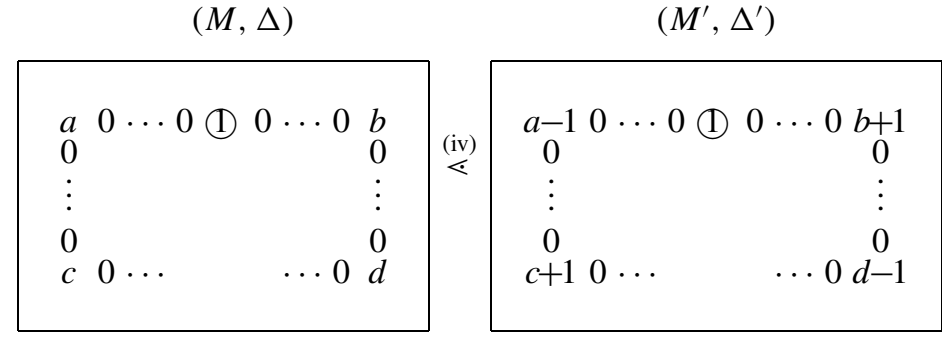

Here $c$ must not be circled, and there must be no circled element weakly southeast of $c$. The move does not change the circled positions.

(v) Suppose, for $t \geq 1$, we have $i_{0}<i_{1}<\cdots<i_{t}$, and $j_{1}>j_{2}>\cdots>j_{t}>j_{0}$, with $\left(i_{1}, j_{1}\right), \ldots,\left(i_{t}, j_{t}\right) \in \Delta, m_{i_{0} j_{0}}>0$, and $m_{i j}=0$ whenever: $\left(i_{0}, j_{0}\right)<(i, j) \leq\left(i_{s}-1, j_{s}-1\right)$ for some $s=1, \ldots, t$. Then define:

$$
\begin{aligned}
M^{\prime} & :=M-E_{i_{0} j_{0}}-E_{i_{1} j_{1}}-\cdots-E_{i_{t} j_{t}}+E_{i_{0} j_{1}}+E_{i_{t} j_{0}} \\
\Delta^{\prime} & :=D \backslash\left\{\left(i_{0}, j_{0}\right),\left(i_{1}, j_{1}\right), \ldots,\left(i_{t}, j_{t}\right)\right\} \cup\left\{\left(i_{0}, j_{1}\right),\left(i_{t}, j_{0}\right)\right\}
\end{aligned}
$$

$(M, \Delta)$

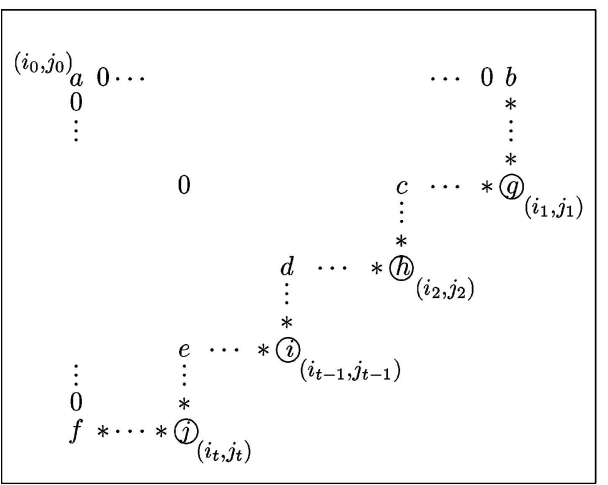

$\left(M^{\prime}, \Delta^{\prime}\right)$

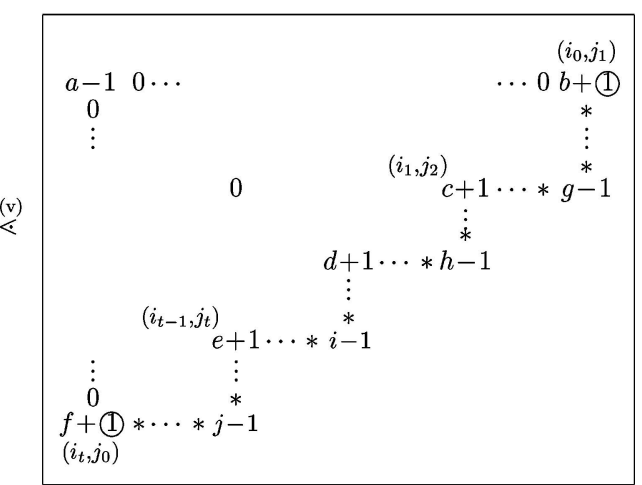

Here $m+$ (1) means $m+1$ circled, and $*$ means a value $m_{i j} \geq 0$, which is unchanged by the move. Note that, in contrast to moves (i)-(iv), we have $\Delta^{\prime}<\Delta$.

\subsection{Strategy of proof}

We will prove the Rank and Move Theorems for triple flags by means of three "chain lemmas." 
Lemma $2 \quad(M, \Delta) \stackrel{\text { mv }}{\leq}\left(M^{\prime}, \Delta^{\prime}\right) \Rightarrow(M, \Delta) \stackrel{\operatorname{deg}}{\leq}\left(M^{\prime}, \Delta^{\prime}\right)$

For $(M, \Delta) \stackrel{\text { mv }}{\leftarrow}\left(M^{\prime}, \Delta^{\prime}\right)^{\prime}$, we will give an explicit degeneration of $\left(M^{\prime}, \Delta^{\prime}\right)$ to $(M, \Delta)$.

Lemma $3 \quad(M, \Delta) \stackrel{\text { deg }}{\leq}\left(M^{\prime}, \Delta^{\prime}\right)^{\prime} \Rightarrow(M, \Delta) \stackrel{\text { rk }}{\leq}\left(M^{\prime}, \Delta^{\prime}\right)$

This will follow from general principles of algebraic geometry.

Lemma $4 \quad(M, \Delta) \stackrel{\text { rk }}{\leq}\left(M^{\prime}, \Delta^{\prime}\right) \Rightarrow(M, \Delta) \stackrel{\text { mv }}{\leq}\left(M^{\prime}, \Delta^{\prime}\right)$

This is a purely combinatorial result. Given $(M, \Delta) \stackrel{\text { rk }}{\leq}\left(M^{\prime}, \Delta^{\prime}\right)$, we construct $(\tilde{M}, \tilde{\Delta})$ with $(M, \Delta) \lessdot(\tilde{M} \tilde{\Delta}) \leq\left(M^{\prime}, \Delta^{\prime}\right)$.

\section{Proof of Theorem 1}

In order to prepare and illuminate the proofs of Lemmas 2-4 for triple flags, we give the precisely analogous arguments for the classical case of two flags, thereby proving Theorem 1.

Lemma $5 \quad M \stackrel{\text { mv }}{\leq} M^{\prime} \Rightarrow M \stackrel{\text { deg }}{\leq} M^{\prime}$

Proof: Given $M \stackrel{\mathrm{mv}}{\leftarrow} M^{\prime}$, it suffices to find a one-parameter algebraic family of double flags (B. $\left.(\tau), C_{\bullet}(\tau)\right)$, indexed by $\tau \in \mathbf{k}$, such that:

$$
\begin{aligned}
& \left(B_{\bullet}(\tau), C_{\bullet}(\tau)\right) \in F_{M^{\prime}} \text { for } \tau \neq 0, \\
& \left(B_{\bullet}(0), C_{\bullet}(0)\right) \in F_{M} .
\end{aligned}
$$

Consider a basis of $V$ indexed according to $M=\left(m_{i j}\right)$ as:

$$
V=\left\langle e_{i j k} \mid(i, j) \in[1, q] \times[1, r], 1 \leq k \leq m_{i j}\right\rangle,
$$

and define a set of vectors indexed according to $M^{\prime}=\left(m_{i j}^{\prime}\right)$,

$$
\left\{v_{i j k}(\tau) \mid(i, j) \in[1, q] \times[1, r], 1 \leq k \leq m_{i j}^{\prime}\right\}
$$

as follows. Let us use the symbol $e_{i j \max }$ to mean that the third subscript in $e_{i j k}$ has as large a value as possible, namely $\max =m_{i j}$; and similarly $v_{i j \max }$ means $\max =m_{i j}^{\prime}$. Now let:

$$
\begin{aligned}
v_{i_{0} j_{1} \max }(\tau) & :=e_{i_{0} j_{0} \max }+\tau e_{i_{1} j_{1} \max } \\
v_{i_{1} j_{0} \max }(\tau) & :=e_{i_{0} j_{0} \max } \\
v_{i j k}(\tau) & :=e_{i j k} \quad \text { otherwise. }
\end{aligned}
$$

For $\tau \neq 0$, let $B_{i}(\tau):=\left\langle v_{i^{\prime} j k} \mid i^{\prime} \leq i\right\rangle, C_{j}(\tau):=\left\langle v_{i j^{\prime} k} \mid j^{\prime} \leq j\right\rangle$. For $\tau \neq 0$, the set $\left\{v_{i j k}\right\}$ forms a basis of $V$, and with respect to this basis $\left(B_{\bullet}(\tau), C_{\bullet}(\tau)\right) \in F_{M^{\prime}}$. 
Now define $B_{i}(0):=\lim _{\tau \rightarrow 0} B_{i}(\tau)$ and $C_{j}(0):=\lim _{\tau \rightarrow 0} C_{j}(\tau)$, the limits in the Zariski topology. ${ }^{3}$ We proceed to evaluate these limits, showing that, with respect to the basis $\left\langle e_{i j k}\right\rangle$, we have $\left(B_{\bullet}(0), C_{\bullet}(0)\right) \in F_{M}$.

As $\tau \rightarrow 0$, the vectors $\left\{v_{i^{\prime} j k}(\tau) \mid i^{\prime} \leq i_{0}\right\}$ remain linearly independent, and $v_{i_{0} j_{1} \max }(\tau) \rightarrow$ $e_{i_{0} j_{0} \max }$, so we have:

$$
B_{i_{0}}(0)=\left\langle v_{i^{\prime} j k}(0) \mid i^{\prime} \leq i_{0}\right\rangle=\left\langle e_{i^{\prime} j k} \mid i^{\prime} \leq i_{0}\right\rangle
$$

Furthermore, we can take linear combinations of basis vectors to find, for arbitrary $\tau \neq 0$ :

$$
\begin{aligned}
B_{i_{1}}(\tau)= & \left\langle e_{i_{0} j_{0} \max }+\tau e_{i_{1} j_{1} \max }, e_{i_{0} j_{0} \max }\right\rangle \\
& \oplus\left\langle v_{i^{\prime} j k} \mid i^{\prime} \leq i_{1},(i, j, k) \neq\left(i_{0}, j_{1}, m_{i_{0} j_{1}}^{\prime}\right),\left(i_{1}, j_{0}, m_{i_{1}, j_{0}}^{\prime}\right)\right\rangle \\
= & \left\langle e_{i_{0} j_{0} \max }, e_{i_{1} j_{1} \max }\right\rangle \\
& \oplus\left\langle e_{i^{\prime} j k} \mid i^{\prime} \leq i_{0},(i, j, k) \neq\left(i_{0}, j_{0}, m_{i_{0} j_{0}}\right),\left(i_{1}, j_{1}, m_{i_{1}, j_{1}}\right)\right\rangle \\
= & \left\langle e_{i^{\prime} j k} \mid i^{\prime} \leq i_{1}\right\rangle .
\end{aligned}
$$

Since the final basis is constant with respect to $\tau$, the limit space $B_{i_{1}}(0)$ exists and has the same basis. Similarly for any $i, j$ we have $B_{i}(0)=\left\langle e_{i^{\prime} j k} \mid i^{\prime} \leq i\right\rangle, C_{j}(0)=\left\langle e_{i j^{\prime} k}\right|$ $\left.j \leq j^{\prime}\right\rangle$.

Lemma $6 \quad M \stackrel{\operatorname{deg}}{\leq} M^{\prime} \Rightarrow M \stackrel{\text { rk }}{\leq} M^{\prime}$

Proof: Since $M \stackrel{\operatorname{deg}}{\leq} M^{\prime}$, there exists an algebraic family with $\left(B_{\bullet}(\tau), C_{\bullet}(\tau)\right) \in F_{M^{\prime}}$ for $\tau \neq 0$, and $\left(B_{\bullet}(0), C_{\bullet}(0)\right) \in F_{M}$.

For any fixed $d$, the condition $\operatorname{dim}\left(B_{i} \cap C_{j}\right) \geq d$ defines a closed subvariety of $\operatorname{Flag}(\mathbf{b}) \times$ Flag(c) (cut out by the vanishing of certain determinants in the homogeneous coordinates of the flag varieties). Hence if the generic elements of the family satisfy $r_{i j}\left(M^{\prime}\right) \geq d$, then so does the limit element at $\tau=0$. That is, the rank numbers $r_{i j}\left(M^{\prime}\right)$ can only get larger upon degeneration.

Lemma $7 \quad M \stackrel{\text { rk }}{\leq} M^{\prime} \Rightarrow M \stackrel{\text { mv }}{\leq} M^{\prime}$.

In fact, if $M \stackrel{\mathrm{rk}}{<} \bar{M}^{\prime}$, then we can perform a simple move on $M$ to obtain a matrix $\tilde{M}$ satisfying

$$
M \stackrel{\mathrm{mv}}{\lessdot} \stackrel{\mathrm{rk}}{\leq} M^{\prime} .
$$

Proof: Denote $M=\left(m_{i j}\right), r_{i j}=r_{i j}(M), \quad M^{\prime}=\left(m_{i j}^{\prime}\right), r_{i j}^{\prime}=r_{i j}\left(M^{\prime}\right)$, so that $r_{i j} \geq r_{i j}^{\prime}$ by assumption. Consider the lexicographically first position $\left(k_{0}, l_{0}\right)$ where the matrices differ: that is, $m_{i j}=m_{i j}^{\prime}$ for $i<k_{0}$ and for $i=k_{0}, j<l_{0}$; and $m_{k_{0} l_{0}}>m_{k_{0} l_{0}}^{\prime}$, making $r_{k_{0} l_{0}}>r_{k_{0} l_{0}}^{\prime}$. Consider a rectangle $\left[k_{0}, k_{1}\right] \times\left[l_{0}, l_{1}\right]$ as large as possible such that $r_{i j}>r_{i j}^{\prime}$ for $(i, j) \in\left[k_{0}, k_{1}-1\right] \times\left[l_{0}, l_{1}-1\right]$. (Such a rectangle is not necessarily unique.)

Claim: $m_{i_{1} j_{1}}>0$ for some $\left(i_{1}, j_{1}\right) \in\left[k_{0}+1, k_{1}\right] \times\left[l_{0}+1, l_{1}\right]$. Otherwise we would have $m_{i j}=0$ for all $(i, j) \in\left[k_{0}+1, k_{1}\right] \times\left[l_{0}+1, l_{1}\right]$. By the maximality of the rectangle, we have $r_{i l_{1}}=r_{i l_{1}}^{\prime}$ and $r_{k_{1} j}=r_{k_{1} j}^{\prime}$ for some $(i, j) \in\left[k_{0}, k_{1}-1\right] \times\left[j_{0}, j_{2}-1\right]$. By the definition 
of the rank numbers and the vanishing of the $m_{k l}$, we have:

$$
r_{k_{1} l_{1}}=r_{i l_{1}}+r_{k_{1} j}-r_{i j}<r_{i l_{1}}^{\prime}+r_{k_{1} j}^{\prime}-r_{i j}^{\prime} \leq r_{k_{1} l_{1}}^{\prime}
$$

This contradiction proves our claim.

We may assume the $\left(i_{1}, j_{1}\right)$ found above is $\leq$-minimal, so that $m_{i j}=0$ for $(i, j) \in$ $\left[k_{0}+1, i_{1}\right] \times\left[l_{0}+1, j_{1}\right],(i, j) \neq\left(i_{1}, j_{1}\right)$. By moving right or down from $\left(k_{0}, l_{0}\right)$ to a position $\left(i_{0}, j_{0}\right)$, we can get:

$$
\begin{aligned}
m_{i_{0} j_{0}}, m_{i_{1} j_{1}}>0, \quad m_{i j} & =0 \quad \text { for }\left(i_{0}, j_{0}\right)<(i, j)<\left(i_{1}, j_{1}\right),(i, j) \neq\left(i_{0}, j_{1}\right),\left(i_{1}, j_{0}\right) \\
r_{i j} & >r_{i j}^{\prime} \quad \text { for }(i, j) \in\left[i_{0}, i_{1}-1\right] \times\left[j_{0}, j_{1}-1\right] .
\end{aligned}
$$

These are all the necessary conditions to perform our simple move: $\tilde{M}:=M-E_{i_{0} j_{0}}-$ $E_{i_{1} j_{1}}+E_{i_{1} j_{0}}+E_{i_{0} j_{1}}$. Denoting $\tilde{r}_{i j}=r_{i j}(\tilde{M})$, we have:

$$
\tilde{r}_{i j}= \begin{cases}r_{i j}-1 \geq r_{i j}^{\prime} & \text { if }(i, j) \in\left[i_{0}, j_{1}-1\right] \times\left[j_{0}, j_{1}-1\right] \\ r_{i j} \geq r_{i j}^{\prime} & \text { otherwise }\end{cases}
$$

showing that $\tilde{M} \stackrel{\text { rk }}{\leq} M^{\prime}$.

Thus, all three orders are identical, denoted $M \leq M^{\prime}$. It only remains to prove that the simple moves give the covers of this order.

Suppose $M \stackrel{R}{\gtrless} M^{\prime}$ for a rectangle $R \underset{\overline{\mathrm{S}}}{=}\left[i_{0}, i_{1}\right] \times\left[j_{0}, j_{1}\right]$, and $M<\tilde{M} \leq M^{\prime}$. Without loss of generality, we may assume $M \stackrel{\mathrm{S}}{\leftarrow} \tilde{M}$ for some other rectangle $S$. Then it is clear that

$$
r_{i j}(M) \geq r_{i j}(\tilde{M}) \geq r_{i j}\left(M^{\prime}\right)
$$

But

$$
r_{i j}(M)= \begin{cases}r_{i j}\left(M^{\prime}\right)+1 & \text { for }(i, j) \in\left[i_{0}, i_{1}-1\right] \times\left[j_{0}, j_{1}-1\right] \\ r_{i j}\left(M^{\prime}\right) & \text { otherwise }\end{cases}
$$

and similarly for $S$. Hence $S \subset R$, but because $m_{i j}=0$ for all $(i, j) \in R$, we must have $S=R$, and $\tilde{M}=M^{\prime}$.

This completes the proof of Theorem 1.

\section{Geometry of decorated matrices}

In this section, we prove the first two chain lemmas for triple flags, the ones involving geometric arguments. We follow the same lines of argument as for two flags. 
Let $(M, \Delta),\left(M^{\prime}, \Delta^{\prime}\right)$ be decorated matrices indexing the orbits in $\mathbb{P}^{n-1} \times \operatorname{Flag}(\mathbf{b}) \times$ Flag(c), and denote as above $M=\left(m_{i j}\right), r_{i j}=r_{i j}(M)$, etc., as well as $r_{\langle i j\rangle}=r_{\langle i j\rangle}(M, \Delta)$, etc. (Later we will also use $\tilde{r}_{i j}=r_{i j}(\tilde{M})$, etc.) Let us first prove the Uncircling Lemma:

Proof of Lemma 1: Suppose we have $\left(i_{0}, j_{0}\right),\left(i_{1}, j_{1}\right) \in S$ with $\left(i_{0}, j_{0}\right)<\left(i_{1}, j_{1}\right)$. Let $S^{\prime}=S \backslash\left\{\left(i_{0}, j_{0}\right)\right\}$, and denote by $F_{M, S}, F_{M, S^{\prime}}$ the GL(V)-orbits defined analogously to $F_{M, \Delta}$. It suffices to show that $F_{M, S}=F_{M, S^{\prime}}$.

Let $\left\langle e_{i j k}\right\rangle$ be a basis of $V$ indexed according to $M$ (that is, $k \leq m_{i j}$ ), and let $B_{i}:=\left\langle e_{i^{\prime} j k}\right|$ $\left.i^{\prime} \leq i\right\rangle, C_{j}:=\left\langle e_{i j^{\prime} k} \mid j^{\prime} \leq j\right\rangle$, and $A:=\left\langle\sum_{(i, j) \in S} e_{i j 1}\right\rangle$. By definition, $\left(A, B_{\bullet}, C_{\bullet}\right) \in F_{M, S}$.

Now let us write the same $\left(A, B_{\bullet}, C_{\bullet}\right)$ in terms of a new basis $\left\langle v_{i j k}\right\rangle$, defined by $v_{i_{1} j_{1} 1}:=$ $e_{i_{1} j_{1} 1}+e_{i_{0} j_{0} 1}$ and $v_{i j k}=e_{i j k}$ otherwise. We have

$$
\begin{aligned}
B_{i_{1}} & =\left\langle e_{i_{0} j_{0} 1}, e_{i_{1} j_{1} 1}\right\rangle \oplus\left\langle e_{i j k} \mid i \leq i_{1},(i, j, k) \neq\left(i_{0}, j_{0}, 1\right),\left(i_{1}, j_{1}, 1\right)\right\rangle \\
& =\left\langle e_{i_{0} j_{0} 1}, e_{i_{1} j_{1} 1}+e_{i_{0} j_{0} 1}\right\rangle \oplus\left\langle e_{i j k} \mid i \leq i_{1},(i, j, k) \neq\left(i_{0}, j_{0}, 1\right),\left(i_{1}, j_{1}, 1\right)\right\rangle \\
& =\left\langle v_{i j k} \mid i \leq i_{1}\right\rangle
\end{aligned}
$$

Similarly $B_{i}=\left\langle v_{i^{\prime} j k} \mid i^{\prime} \leq i\right\rangle$ and $C_{j}=\left\langle v_{i j^{\prime} k} \mid j^{\prime} \leq j\right\rangle$ for all $i, j$. Finally,

$$
\begin{aligned}
A & =\left\langle e_{i_{0} j_{0} 1}+e_{i_{1} j_{1}}+\sum_{(i, j) \in S^{\prime \prime}} e_{i j 1}\right\rangle \\
& =\left\langle v_{i_{1} j_{1}}+\sum_{(i, j) \in S^{\prime \prime}} v_{i j 1}\right\rangle=\left\langle\sum_{(i, j) \in S^{\prime}} v_{i j 1}\right\rangle,
\end{aligned}
$$

where $S^{\prime \prime}=S \backslash\left\{\left(i_{0}, j_{0}\right),\left(i_{1}, j_{1}\right)\right\}$. Hence $\left(A, B_{\bullet}, C_{\bullet}\right) \in F_{M, S^{\prime}}$, proving the Lemma.

Proof of Lemma 2: We follow the method in the proof of Lemma 5. For each type of move $(M, \Delta) \lessdot\left(M^{\prime}, \Delta^{\prime}\right)$ listed in Section 2.3, we start with a basis $V=\left\langle e_{i j k} \mid k \leq m_{i j}\right\rangle$ indexed according to $M$; then define another set of vectors $\left\langle v_{i j k}(\tau) \mid k \leq m_{i j}^{\prime}\right\rangle$ indexed according to $M^{\prime}$ which forms a basis when $\tau \neq 0$.

We then define a family of triple flags for $\tau \neq 0$ by:

$$
\begin{aligned}
B_{i}(\tau) & :=\left\langle v_{i^{\prime} j k}(\tau) \mid i^{\prime} \leq i\right\rangle, C_{j}(\tau):=\left\langle v_{i j^{\prime} k}(\tau) \mid j^{\prime} \leq j\right\rangle, \\
A(\tau) & :=\left\langle\sum_{(i, j) \in \Delta^{\prime}} v_{i j 1}(\tau)\right\rangle
\end{aligned}
$$

so that by definition $\left(A(\tau), B_{\bullet}(\tau), C_{\bullet}(\tau)\right) \in F_{M^{\prime}, \Delta^{\prime}}$ with respect to the basis $\left\langle v_{i j k}(\tau)\right\rangle$. Furthermore, it will be clear that as $\tau \rightarrow 0$, we have

$$
\left(A(\tau), B_{\bullet}(\tau), C_{\bullet}(\tau)\right) \rightarrow\left(A(0), B_{\bullet}(0), C_{\bullet}(0)\right),
$$


where

$$
\begin{aligned}
B_{i}(0) & :=\left\langle e_{i^{\prime} j k} \mid i^{\prime} \leq i\right\rangle, \quad C_{j}(0):=\left\langle e_{i j^{\prime} k} \mid j^{\prime} \leq j\right\rangle . \\
A(0) & :=\left\langle\sum_{(i, j) \in \Delta} e_{i j 1}\right\rangle .
\end{aligned}
$$

This shows that $\lim _{\tau \rightarrow 0}\left(A(\tau), B_{\bullet}(\tau), C_{\bullet}(\tau)\right) \in F_{M, \Delta}$, and proves the Lemma. We will give details only in the last, most complicated case.

(i) Let $S:=\left\{(i, j) \in \Delta \mid(i, j)<\left(i_{1}, j_{1}\right)\right\}$. Now define: $v_{i_{1} j_{1} 1}:=\tau e_{i_{1} j_{1} 1}+\sum_{(i, j) \in S} e_{i j 1}$ and $v_{i j k}:=e_{i j k}$ otherwise. (In each of the following cases, we implicitly define $v_{i j k}:=e_{i j k}$ for those $(i, j, k)$ which are not otherwise specified.)

(ii) Since $\Delta=\Delta^{\prime}$, the line $A$ is unchanged in the move. Thus we may take $v_{i j k}$ exactly as in the proof of Lemma 5 .

(iii) Let $S:=\left\{(i, j) \in \Delta \mid(i, j)<\left(i_{0}, j_{1}\right)\right\}$. For (iii)(a), define: $v_{i_{1} j_{0} \max }:=e_{i_{0} j_{0}}$ and $v_{i_{0} j_{1} 1}:=\tau e_{i_{1} j_{1} \max }+\sum_{(i, j) \in S} e_{i j 1}$. Transpose for (iii)(b).

(iv)(a) Let $S:=\left\{(i, j) \in \Delta \mid(i, j)<\left(i_{2}, j_{0}\right)\right\}$. Now define: $v_{i_{1} j_{2} \max }:=e_{i_{2} j_{2} 1}+\tau e_{i_{1} j_{1} \max }$, $v_{i_{0} j_{1} \max }:=e_{i_{0} j_{0} 1}+\tau e_{i_{1} j_{1} \max }$, and $v_{i_{2} j_{0} 1}:=\sum_{(i, j) \in S} e_{i j 1}$.

(iv)(bc) Same as (ii).

(v) Let $S:=\Delta \backslash\left\{\left(i_{1}, j_{1}\right), \ldots,\left(i_{t}, j_{t}\right)\right\}$. Define:

$$
\begin{aligned}
v_{i j 1}:= & \tau e_{i j 1} \quad \text { for }(i, j) \in S ; \quad v_{i_{s} j_{s} k}:=e_{i_{s}, j_{s}, k+1} \quad \text { for } s=1,2, \ldots, t \\
v_{i_{0} j_{1} 1}:= & e_{i_{0} j_{0} \max }+\tau \sum_{s=1}^{t} e_{i_{s} j_{s} 1}, \quad v_{i_{0} j_{1} k}:=e_{i_{0}, j_{1}, k-1} \quad \text { for } k>1 \\
v_{i_{t} j_{0} 1}:= & -e_{i_{0} j_{0} \max }, \quad v_{i_{t} j_{0} k}:=e_{i_{t}, j_{0}, k-1} \quad \text { for } k>1 \\
v_{i_{s} j_{s+1} \max }:= & e_{i_{0} j_{0} \max }+\tau^{2} e_{i_{1} j_{1} 1}+\tau^{2} e_{i_{2} j_{2} 1}+\cdots+\tau^{2} e_{i_{s} j_{s} 1} \\
& +\tau e_{i_{s+1} j_{s+1} 1}+\cdots+\tau e_{i_{t} j_{t} 1} \quad \text { for } s=1,2, \ldots, t-1 .
\end{aligned}
$$

The crucial part of the transition matrix between $\left\langle v_{i j k}\right\rangle$ and $\left\langle e_{i j k}\right\rangle$, containing all the nonzero "non-diagonal" coefficients, is:

\begin{tabular}{ccccccc}
\hline & $v_{i_{0} j_{1} 1}$ & $v_{i_{1} j_{2} \max }$ & $v_{i_{2} j_{3} \max }$ & $\cdots$ & $v_{i_{t-1} j_{t} \max }$ & $v_{i_{t} j_{0} 1}$ \\
\hline$e_{i_{0} j_{0} \max }$ & 1 & 1 & 1 & $\cdots$ & 1 & -1 \\
$e_{i_{1} j_{1} 1}$ & $\tau$ & $\tau^{2}$ & $\tau^{2}$ & $\cdots$ & $\tau^{2}$ & 0 \\
$e_{i_{2} j_{2} 1}$ & $\tau$ & $\tau$ & $\tau^{2}$ & $\cdots$ & $\tau^{2}$ & 0 \\
$\vdots$ & $\vdots$ & $\vdots$ & $\vdots$ & & $\vdots$ & $\vdots$ \\
$e_{i_{t-1} j_{t-1} 1}$ & $\tau$ & $\tau$ & $\tau$ & $\cdots$ & $\tau^{2}$ & 0 \\
$e_{i_{t} j_{t} 1}$ & $\tau$ & $\tau$ & $\tau$ & $\cdots$ & $\tau$ & 0
\end{tabular}


Let us focus on these basis vectors, suppressing all other vectors with ellipsis marks .... We compute, as $\tau \rightarrow 0$ :

$$
\begin{aligned}
B_{i_{0}}(\tau) & =\left\langle v_{i_{0} j_{1} 1}, \ldots\right\rangle \rightarrow\left\langle e_{i_{0} j_{0} \max }, \ldots\right\rangle, \\
B_{i_{1}}(\tau) & =\left\langle v_{i_{0} j_{1} 1}, v_{i_{1} j_{2} \max }, \ldots\right\rangle=\left\langle v_{i_{0} j_{1} 1}, \tau^{-1}\left(v_{i_{0} j_{1} 1}-v_{i_{1} j_{2} \max }\right), \ldots\right\rangle \\
& =\left\langle v_{i_{0} j_{1} 1},(1-\tau) e_{i_{1} j_{1} 1}, \ldots\right\rangle \rightarrow\left\langle e_{i_{0} j_{0} \max }, e_{i_{1} j_{1}}, \ldots\right\rangle,
\end{aligned}
$$

and similarly for the other $B_{i}$. Also:

$$
\begin{aligned}
C_{j_{0}}(\tau) & =\left\langle v_{i_{t} j_{0} 1}, \ldots\right\rangle \rightarrow\left\langle e_{i_{0} j_{0} \max }, \ldots\right\rangle, \\
C_{j_{t}}(\tau) & =\left\langle v_{i_{t} j_{0} 1}, v_{i_{t-1} j_{t} \max }, \ldots\right\rangle=\left\langle-v_{i_{t} j_{0} 1}, \tau^{-1}\left(v_{i_{t-1} j_{t} \max }+v_{i_{t} j_{0} 1}\right), \ldots\right\rangle \\
& =\left\langle v_{i_{t} j_{0} 1}, \tau e_{i_{1} j_{1} 1}+\cdots+\tau e_{i_{t-1} j_{t-1} 1}+e_{i_{t} j_{t}}, \ldots\right\rangle \rightarrow\left\langle e_{i_{0} j_{0} \max }, e_{i_{t} j_{t}}, \ldots\right\rangle,
\end{aligned}
$$

and similarly for the other $C_{j}$. Finally,

$$
\begin{aligned}
A(\tau) & =\left\langle v_{i_{0} j_{1} 1}+v_{i_{t} j_{0} 1}+\sum_{(i, j) \in S} v_{i j 1}\right\rangle \\
& =\left\langle\tau e_{i_{1} j_{1}}+\cdots+\tau e_{i_{t} j_{t} 1}+\sum_{(i, j) \in S} \tau e_{i j 1}\right\rangle \\
& =\left\langle e_{i_{1} j_{1}}+\cdots+e_{i_{t} j_{t} 1}+\sum_{(i, j) \in S} e_{i j 1}\right\rangle .
\end{aligned}
$$

In each case the $\tau \rightarrow 0$ limit is the desired subspace, an element of $F_{M, \Delta}$ with respect to the basis $\left\langle e_{i j k}\right\rangle$.

Proof of Lemma 3: We must show that $r_{\langle i j\rangle}\left(A, B_{\bullet}, C_{\bullet}\right) \geq d$ is a closed condition on $\mathbb{P}(V) \times \operatorname{Flag}(\mathbf{b}) \times \operatorname{Flag}(\mathbf{c})$. This is clear if $i=0$ or $j=0$. Given $(i, j) \in[1, q] \times[1, r]$ and a triple flag $X=\left(A, B_{\bullet}, C_{\bullet}\right)$, define the linear map $\phi_{i j}^{X}: B_{i} \times C_{j} \rightarrow V / A$ by $\left(v_{1}, v_{2}\right) \mapsto v_{1}+v_{2} \bmod A$. Then $\phi_{i j}^{X}$ depends algebraically on $X$ : indeed, we may write $\phi_{i j}^{X}$ in coordinates as a matrix of size $(n-1) \times\left(b_{i}+c_{j}\right)$ with entries depending polynomially on the homogeneous coordinates of $X$. Thus $\operatorname{dim} \operatorname{Ker}\left(\phi_{i j}^{X}\right) \geq d$ is a closed condition on $X$. However,

$$
\begin{aligned}
\operatorname{dim} \operatorname{Ker}\left(\phi_{i j}^{X}\right) & =\operatorname{dim} B_{i}+\operatorname{dim} C_{j}-\operatorname{rank}\left(\phi_{i j}^{X}\right) \\
& =\operatorname{dim} B_{i}+\operatorname{dim} C_{j}-\operatorname{dim}\left(\left(B_{i}+C_{j}\right) / A\right) \\
& =\operatorname{dim} B_{i}+\operatorname{dim} C_{j}-\operatorname{dim}\left(B_{i}+C_{j}\right)+\operatorname{dim}\left(A \cap\left(B_{i}+C_{j}\right)\right) \\
& =\operatorname{dim}\left(B_{i} \cap C_{j}\right)+\operatorname{dim}\left(A \cap\left(B_{i}+C_{j}\right)\right) \\
& =r_{\langle i j\rangle}\left(A, B_{\bullet}, C_{\bullet}\right)
\end{aligned}
$$




\section{Combinatorics of decorated matrices}

To prove the Rank and Move Theorems, it only remains to show Lemma 4.

Thus, assume we are given $(M, \Delta) \stackrel{\text { rk }}{<}\left(M^{\prime}, \Delta^{\prime}\right)$. We wish to show that we can perform one of the moves (i)-(v) on $(M, \Delta)$ to obtain a decorated matrix $(\tilde{M}, \tilde{\Delta})$ satisfying:

$$
(M, \Delta) \stackrel{\mathrm{mv}}{\leftarrow}(\tilde{M}, \tilde{\Delta}) \stackrel{\mathrm{rk}}{\leq}\left(M^{\prime}, \Delta^{\prime}\right) .
$$

We give an algorithm producing $(\tilde{M}, \tilde{\Delta})$.

For cases (i)-(v)(a), we assume $\Delta \leq \Delta^{\prime}$. This is equivalent to $\delta_{i j}(\Delta) \geq \delta_{i j}\left(\Delta^{\prime}\right)$ for all $(i, j)$. Note that if $(\tilde{M}, \tilde{\Delta})$ is any decorated matrix satisfying $\tilde{M} \leq M^{\prime}$ and $\tilde{\Delta} \leq \Delta^{\prime}$, then $(\tilde{M}, \tilde{\Delta}) \stackrel{\text { rk }}{\leq}\left(M^{\prime}, \Delta^{\prime}\right)$.

Case (i). Assume $M=M^{\prime}$. Then $\Delta<\Delta^{\prime}$, and we may choose a minimal $\left(i_{1}, j_{1}\right)$ with $\left(i_{1}, j_{1}\right) \not \Delta,\left(i_{1}, j_{1}\right) \leq \Delta^{\prime}$. Now we may apply move (i) to the appropriate block $\left[i_{0}, i_{1}\right] \times\left[j_{0}, j_{1}\right]$ of $(M, \Delta)$, obtaining $(\tilde{M}, \tilde{\Delta})$. Since $M=\tilde{M}=M^{\prime}$ and $\Delta<\tilde{\Delta} \leq \Delta^{\prime}$, we have:

$$
(M, \Delta) \stackrel{(\mathrm{i})}{\leftarrow}(\tilde{M}, \tilde{\Delta}) \stackrel{\mathrm{rk}}{\leq}\left(M^{\prime}, \Delta^{\prime}\right) .
$$

For the rest of the cases, we assume $M \neq M^{\prime}$, so that $M \stackrel{\text { rk }}{<} M^{\prime}$ in the rank order for two flags. We may then apply the proof of Lemma 7 to find $\left(i_{0}, j_{0}\right),\left(i_{1}, j_{1}\right)$ such that:

$$
\begin{aligned}
& m_{i_{0} j_{0}}, m_{i_{1} j_{1}}>0, \quad m_{i j}=0 \text { for }\left(i_{0}, i_{1}\right)<(i, j)<\left(i_{1}, j_{1}\right),(i, j) \neq\left(i_{0}, j_{1}\right),\left(i_{1}, j_{0}\right) \\
& \text { and } r_{i j}>r_{i j}^{\prime} \text { for }(i, j) \in R, \text { where } R:=\left[i_{0}, i_{1}-1\right] \times\left[j_{0}, j_{1}-1\right] .
\end{aligned}
$$

Henceforth, in cases (ii)-(v)(a), we will assume as given such positions $\left(i_{0}, j_{0}\right),\left(i_{1}, j_{1}\right)$. Case (ii). Assume $\left(i_{0}, j_{0}\right),\left(i_{1}, j_{1}\right) \notin \Delta$; or $\left(i_{0}, j_{0}\right) \in \Delta, m_{i_{0} j_{0}}>1$.

If $\left(i_{0}, j_{1}\right),\left(i_{1}, j_{0}\right) \in \Delta$, then we may apply move (i) to the block $\left[i_{0}, i_{1}\right] \times\left[j_{0}, j_{1}\right]$ of $(M, \Delta)$, obtaining $\tilde{M}=M, \tilde{\Delta}=\left[\Delta \cup\left\{\left(i_{1}, j_{1}\right)\right\}\right]$. Clearly for all $(i, j), \tilde{r}_{i j}=r_{i j} \geq r_{i j}^{\prime}$; and for $(i, j) \notin, \tilde{r}_{\langle i j\rangle}=r_{\langle i j\rangle} \geq r_{\langle i j\rangle}^{\prime}$; while for $(i, j) \in R, \tilde{r}_{\langle i j\rangle}=r_{i j} \geq r_{i j}^{\prime}+1 \geq r_{\langle i j\rangle}^{\prime}$. Thus $(M, \Delta) \stackrel{(\mathrm{i})}{\leftarrow}(\tilde{M}, \tilde{\Delta}) \leq\left(M^{\prime}, \Delta^{\prime}\right)$.

If, on the other hand, $\left(i_{0}, j_{1}\right) \notin \Delta$ or $\left(i_{1}, j_{0}\right) \notin \Delta$, then we may apply move (ii) to the block $\left[i_{0}, i_{1}\right] \times\left[j_{0}, j_{1}\right]$ of $(M, \Delta)$, obtaining $(M, \Delta) \stackrel{\text { (ii) }}{\leftarrow}(\tilde{M}, \tilde{\Delta})$. For any $(i, j)$ we have $\tilde{M} \stackrel{\mathrm{rk}}{\leq} M^{\prime}$ by Lemma 7 , and by assumption $\tilde{\Delta}=\Delta \leq \Delta^{\prime}$, so $(M, \Delta) \stackrel{(\mathrm{i})}{\leftarrow}(\tilde{M}, \tilde{\Delta}) \stackrel{\mathrm{rk}}{\leq}\left(M^{\prime}\right.$, $\left.\Delta^{\prime}\right)$.

Case (iii). (a) Assume $\left(i_{0}, j_{0}\right) \in \Delta, m_{i_{0} j_{0}}=1$, and $\left(i_{0}, j_{1}\right) \leq \Delta^{\prime}$.

If $m_{i j}>0$, for some $(i, j)$ in the rectangle $\left[1, i_{0}\right] \times\left[j_{0}, j_{1}\right]$ and with $(i, j) \not \Delta \Delta$, we may apply move (i) to some block in this rectangle, obtaining $(M, \Delta) \stackrel{(1)}{\lessdot}(\tilde{M}, \tilde{\Delta})$ with $M=\tilde{M} \stackrel{\text { rk }}{\leq} M^{\prime}$ and $\Delta<\tilde{\Delta} \leq \Delta^{\prime}$. Thus $(M, \Delta) \stackrel{(\mathrm{i})}{\leftarrow}(\tilde{M}, \tilde{\Delta}) \stackrel{\text { rk }}{\leq}\left(M^{\prime}, \Delta^{\prime}\right)$.

Otherwise $m_{i j}=0$ for all $(i, j)$ in $\left[1, i_{0}\right] \times\left[j_{0}, j_{1}\right]$ with $(i, j) \not \leq \Delta$, so we have all the conditions necessary to apply move (iii)(a) to the block $\left[i_{0}, i_{1}\right] \times\left[j_{0}, j_{1}\right]$ of $(M, \Delta)$, 
obtaining $(M, \Delta) \stackrel{\text { (iii) }}{\leftarrow}(\tilde{M}, \tilde{\Delta})$. As before, $\tilde{M} \stackrel{\text { rk }}{\leq} M^{\prime}$ by Lemma 7 , and $\tilde{\Delta} \leq \Delta \cup\left\{\left(i_{0}, j_{1}\right)\right\}$ $\leq \Delta^{\prime}$ by assumption, so $(M, \Delta) \stackrel{\text { (iii) }}{<}(\tilde{M}, \tilde{\Delta}) \stackrel{\text { rk }}{\leq}\left(M^{\prime}, \Delta^{\prime}\right)$.

Case $(i i i)(b)$. Assume $\left(i_{0}, j_{0}\right) \in \Delta, m_{i_{0} j_{0}}=1$, and $\left(i_{1}, j_{0}\right) \leq \Delta^{\prime}$. We can repeat the arguments of the previous case with rows and columns transposed.

Case $(i v)(a)$. Assume $\left(i_{0}, j_{0}\right) \in \Delta, m_{i_{0} j_{0}}=1$, and $\left(i_{1}, j_{0}\right),\left(i_{0}, j_{1}\right) \not \leq \Delta^{\prime}$; and furthermore that $m_{i j}=m_{i j}^{\prime}$ for $i \leq i_{0}$ and for $i=i_{0}, j<j_{0}$. (In terms of the proof of Lemma 7, the last condition means $\left(i_{0}, j_{0}\right)=\left(k_{0}, l_{0}\right)$.)

Our assumptions imply:

$$
\Delta^{\prime} \leq\left\{\left(i_{0}-1, r\right),\left(q, j_{0}-1\right),\left(i_{1}-1, j_{1}-1\right)\right\}
$$

Since $\left(i_{0}, j_{0}\right) \in \Delta \leq \Delta^{\prime}$, there must exist $\left(s_{1}, t_{1}\right) \in \Delta^{\prime} \cap R$.

Claim: $r_{i, j_{0}-1}>r_{i, j_{0}-1}^{\prime}$ for $i \in\left[s_{1}, i_{1}-1\right]$. Otherwise, if $r_{i, j_{0}-1}=r_{i, j_{0}-1}^{\prime}$ for some $i \in\left[s_{1}, i_{1}-1\right]$, we would have:

$$
\begin{aligned}
r_{i t_{1}} & =r_{i, j_{0}-1}+r_{i_{0}-1, t_{1}}-r_{i_{0}-1, j_{0}-1}+1 \\
& \leq r_{i, j_{0}-1}^{\prime}+r_{i_{0}-1, t_{1}}^{\prime}-r_{i_{0}-1, j_{0}-1}^{\prime}+\sum_{\left(i_{0}, j_{0}\right) \leq(k, l) \leq\left(i, t_{1}\right)} m_{k l}^{\prime} \\
& =r_{i t_{1}}^{\prime} .
\end{aligned}
$$

This contradicts $r_{i j}>r_{i j}^{\prime}$ within $R$, establishing the claim.

Now take a rectangle $S:=\left[s_{0}, i_{1}-1\right] \times\left[t_{0}, j_{0}-1\right]$ as large as possible such that $r_{i j}>r_{i j}^{\prime}$ for $(i, j) \in S$. Further, take $\left(s_{0}, t_{0}\right)$ to be lexicographically minimal with the above property, so that $s_{0} \leq s_{1}$ by the above Claim.

Claim: There exists $\left(i_{2}, j_{2}\right) \in S$ such that $m_{i_{2} j_{2}}>0$. Otherwise, we would have $m_{i j}=0$ within $S$. By the maximality of $S$, there exists $(i, j) \in S$ so that $r_{i, t_{0}-1}=r_{i, t_{0}-1}^{\prime}$ and $r_{s_{0}-1, j}=r_{s_{0}-1, j}^{\prime}$. Then

$$
r_{i j}=r_{s_{0}-1, j}+r_{i, t_{0}-1}-r_{s_{0}-1, t_{0}-1} \leq r_{s_{0}-1, j}^{\prime}+r_{i, t_{0}-1}^{\prime}-r_{s_{0}-1, t_{0}-1}^{\prime} \leq r_{i j}^{\prime}
$$

This contradicts $r_{i j}>r_{i j}^{\prime}$ within $S$, establishing the claim.

Thus, we may choose $\left(i_{2}, j_{2}\right)$ in $S$ with $m_{i_{2} j_{2}}>0$ and $m_{i j}=0$ for $\left(i_{2}, j_{2}\right)<(i, j) \leq$ $\left(i_{1}-1, j_{0}-1\right)$. In fact, choose $\left(i_{2}, j_{2}\right)$ to be as northeast as possible with these properties, so that $m_{i j}=0$ for $(i, j) \in\left[s_{0}, i_{2}-1\right] \times\left[j_{2}+1, j_{0}-1\right]$. If $\left(i_{2}, j_{2}\right) \notin \Delta$ or $m_{i_{2} j_{2}}>1$, we have all the necessary conditions to apply move (ii) to the block $\left[i_{2}, i_{1}\right] \times\left[j_{2}, j_{1}\right]$ or $\left[i_{2}, i_{1}\right] \times\left[j_{2}, j_{0}\right]$ of $(M, \Delta)$, and finish as in case (ii) above, obtaining

$$
(M, \Delta) \stackrel{\text { (ii) }}{\leftarrow}(\tilde{M}, \tilde{\Delta}) \stackrel{\text { rk }}{\leq}\left(M^{\prime}, \Delta^{\prime}\right) .
$$

If $m_{i j}>0$ for some $(i, j)$ in the rectangle $\left[1, s_{0}-1\right] \times\left[j_{2}, j_{0}-1\right]$ with $(i, j) \not \leq \Delta$, then we may apply move (i) to some block within this rectangle. Since the southeast corner 
$\left(s_{0}-1, j_{0}-1\right) \leq\left(s_{1}, t_{1}\right) \in \Delta^{\prime}$, we obtain:

$$
(M, \Delta) \stackrel{(\mathrm{i})}{<}(\tilde{M}, \tilde{\Delta}) \stackrel{\text { rk }}{\leq}\left(M^{\prime}, \Delta^{\prime}\right) \text {. }
$$

Thus, we have reduced to the case where $\left(i_{2}, j_{2}\right) \in \Delta, m_{i_{2} j_{2}}=1$, and $m_{i j}=0$ or $(i, j) \in \Delta$ for all $(i, j) \in\left[1, i_{2}-1\right] \times\left[j_{2}, j_{0}-1\right]$.

If $m_{i_{1} j_{0}}>0$, we may apply move (iii) to the block $\left[i_{2}, i_{1}\right] \times\left[j_{2}, j_{0}\right]$ of $(M, \Delta)$. Then clearly $\tilde{r}_{i j} \geq r_{i j}^{\prime}$, since the block lies inside $S$. For $(i, j)$ outside $\left[i_{0}, i_{2}-1\right] \times\left[j_{2}, j_{0}-1\right]$, we have

$$
(i+1, j+1) \leq \tilde{\Delta} \Leftrightarrow(i+1, j+1) \leq \Delta \Rightarrow(i+1, j+1) \leq \Delta^{\prime},
$$

so

$$
\tilde{r}_{\langle i j\rangle}=\tilde{r}_{i j}+\delta_{i j}(\tilde{\Delta})=\tilde{r}_{i j}+\delta_{i j}(\Delta) \geq r_{i j}^{\prime}+\delta_{i j}\left(\Delta^{\prime}\right)=r_{\langle i j\rangle}^{\prime}
$$

For $(i, j) \in\left[i_{0}, s_{0}-1\right] \times\left[j_{2}, j_{0}-1\right]$, we have

$$
(i+1, j+1) \leq\left(s_{0}, j_{0}\right) \leq\left(s_{1}, t_{1}\right) \in \Delta^{\prime}
$$

and $\tilde{r}_{i j}=r_{i j}$, so $\tilde{r}_{\langle i j\rangle} \geq r_{i j} \geq r_{i j}^{\prime}=r_{\langle i j\rangle}^{\prime}$. For $(i, j) \in\left[s_{0}, i_{2}-1\right] \times\left[j_{2}, j_{0}-1\right] \subset S$, we have $\tilde{r}_{\langle i j\rangle} \geq \tilde{r}_{i j}=r_{i j} \geq r_{i j}^{\prime}+1 \geq r_{\langle i j\rangle}^{\prime}$. Therefore

$$
(M, \Delta) \stackrel{\text { (iii) }}{<}(\tilde{M}, \tilde{\Delta}) \stackrel{\text { rk }}{\leq}\left(M^{\prime}, \Delta^{\prime}\right)
$$

Finally, suppose $m_{i_{1} j_{0}}=0$ or $(i, j) \in \Delta$, and as before $\left(i_{2}, j_{2}\right) \in \Delta, m_{i_{2} j_{2}}=1$, and $m_{i j}=0$ for all $(i, j) \in\left[i_{0}+1, i_{2}-1\right] \times\left[j_{2}+1, j_{0}-1\right]$. Then we may apply move (iv)(a) to the rectangle $\left[i_{2}, i_{1}\right] \times\left[j_{2}, j_{1}\right]$ in $(M, \Delta)$. Now, for $(i, j)$ outside the region

$$
\left[i_{2}, i_{1}-1\right] \times\left[j_{2}, j_{1}-1\right] \cup\left[i_{0}, i_{1}-1\right] \times\left[j_{0}, j_{1}-1\right] \subset S \cup R
$$

we have $\tilde{r}_{i j}=r_{i j} \geq r_{i j}^{\prime}$; whereas for $(i, j)$ inside this region we have $\tilde{r}_{i j}=r_{i j}-1 \geq r_{i j}^{\prime}$.

To check $\tilde{r}_{\langle i j\rangle} \geq r_{\langle i j\rangle}^{\prime}$, we repeat the argument we used to show $(M, \Delta) \stackrel{\text { (iii) }}{<}(\tilde{M}, \tilde{\Delta}) \stackrel{\text { rk }}{\leq}$ $\left(M^{\prime}, \Delta^{\prime}\right)$ immediately above. For $(i, j)$ outside the rectangle $\left[i_{0}, i_{2}-1\right] \times\left[j_{2}, j_{0}-1\right]$, we have $(i+1, j+1) \leq \Delta \Leftrightarrow(i+1, j+1) \leq \tilde{\Delta} \Rightarrow(i+1, j+1) \leq \Delta^{\prime}$, so clearly $\tilde{r}_{\langle i j\rangle} \geq r_{\langle i j\rangle}^{\prime}$ as above. Similarly repeat the arguments for $(i, j)$ inside $\left[i_{0}, i_{2}-1\right] \times\left[j_{2}, j_{0}-1\right]$, to obtain

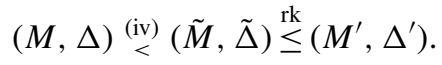

Case $(i v)(b c)$. Assume $\left(i_{0}, j_{0}\right) \in \Delta, m_{i_{0} j_{0}}=1$, and $\left(i_{1}, j_{0}\right),\left(i_{0}, j_{1}\right) \not \leq \Delta^{\prime}$; but that $m_{i j} \neq$ $m_{i j}^{\prime}$ for some $i \leq i_{0}$ or for $i=i_{0}, j<j_{0}$. From the proof of Lemma 7, the last condition means that there exists $k_{0}<i_{0}$ with $m_{k_{0} j_{0}}>0$, or there exists $l_{0}<j_{0}$ with $m_{i_{0} l_{0}}>1$. Assume the first alternative (the other one being merely a transpose). 
By increasing $k_{0}$ if necessary, we may assume that $m_{i j}=0$ for $(i, j) \in\left[k_{0}, i_{1}\right] \times\left[j_{0}, j_{1}\right]$ except that definitely $m_{i j}>0$ for $(i, j)=\left(k_{0}, j_{0}\right),\left(i_{0}, j_{0}\right),\left(i_{1}, j_{1}\right)$, and possibly $m_{i j}>0$ for $(i, j)=\left(i_{1}, j_{0}\right),\left(i_{0}, j_{1}\right)$ and for $i=k_{0}$.

Suppose $m_{i_{0} j_{1}}>0$. If $m_{k_{0} j}>0$ for $j_{0}<j<j_{1}$; or if $m_{k_{0} j_{0}}>0$ and $\left(k_{0}, j_{1}\right) \notin \Delta$; then we may apply move (ii) to the rectangle $\left[k_{0}, i_{0}\right] \times\left[j, j_{1}\right]\left[k_{0}, i_{0}\right] \times\left[j_{0}, j_{1}\right]$, and finish as in case (ii): $(M, \Delta) \stackrel{\text { (ii) }}{\leftarrow}(\tilde{M}, \tilde{\Delta}) \stackrel{\text { rk }}{\leq}\left(M^{\prime}, \Delta^{\prime}\right)$. Otherwise, if $m_{k_{0} j_{0}}>0, m_{k_{0} j}=0$ for $j_{0}<j<j_{1}$, and $\left(k_{0}, j_{1}\right) \in \Delta$; then apply move (i) to $\left[k_{0}, i_{0}\right] \times\left[j_{0}, j_{1}\right]$ to get $\tilde{\Delta}=\left[\Delta \cup\left\{\left(i_{0}, j_{1}\right)\right\}\right]$, yielding $(M, \Delta) \stackrel{(\mathrm{i})}{\leftarrow}(\tilde{M}, \tilde{\Delta}) \stackrel{\text { rk }}{\leq}\left(M^{\prime}, \Delta^{\prime}\right)$.

Now suppose $m_{i_{0} j_{1}}=0$. If $m_{k_{0} j}>0$ for $j_{0}<j<j_{1}$; then we may apply move (ii) to the rectangle $\left[k_{0}, i_{1}\right] \times\left[j, j_{1}\right]:(M, \Delta) \stackrel{(\mathrm{ii})}{\leftarrow}(\tilde{M}, \tilde{\Delta}) \stackrel{\text { rk }}{\leq}\left(M^{\prime}, \Delta^{\prime}\right)$. If $m_{k_{0} j_{1}} \in \Delta$, then we may apply move (iii) to $\left[i_{0}, i_{1}\right] \times\left[j_{0}, j_{1}\right]$, getting $(M, \Delta) \stackrel{\text { (iii) }}{\leftarrow}(\tilde{M}, \tilde{\Delta}) \stackrel{\text { rk }}{\leq}\left(M^{\prime}, \Delta^{\prime}\right)$.

Suppose none of the above cases hold. Then $m_{i_{0} j_{1}}=0, m_{k_{0} j_{1}} \notin \Delta$, and $m_{k_{0} j}=0$ for $j_{0}<j<j_{1}$. Then we finally have the conditions to apply move (iv)(b) to the rectangle $\left[k_{0}, j_{0}\right] \times\left[j_{0}, j_{1}\right]$, and finish by again noting that $\tilde{M} \stackrel{\mathrm{rk}}{\leq} M^{\prime}, \tilde{\Delta}=\Delta \leq \Delta^{\prime}$, so that $(M, \Delta) \stackrel{\text { (iv) }}{\leftarrow}(\tilde{M}, \tilde{\Delta}) \stackrel{\text { rk }}{\leq}\left(M^{\prime}, \Delta^{\prime}\right)$.

Case $(v)(a)$. Assume $\left(i_{1}, j_{1}\right) \in \Delta$. Then perform move (v) on the rectangle $\left[i_{0}, i_{1}\right] \times\left[j_{0}, j_{1}\right]$ to obtain $(M, \Delta) \stackrel{\text { vv) }}{<}(\tilde{M}, \tilde{\Delta})$. We clearly have $\tilde{M} \stackrel{\text { rk }}{\leq} M^{\prime}$ as well as $\tilde{\Delta} \leq \Delta \leq \Delta^{\prime}$. Hence $(\tilde{M}, \tilde{\Delta}) \stackrel{\text { rk }}{\leq}\left(M^{\prime}, \Delta^{\prime}\right)$.

We have now proved the Lemma assuming $\Delta \leq \Delta^{\prime}$. Next assume $\Delta \not \leq \Delta^{\prime}$.

Case $(v)(b)$. Consider any $(i, j)$ with $(i+1, j+1) \leq \Delta$ but $(i+1, j+1) \not \leq \Delta^{\prime}$. Then we have $r_{i j}+0=r_{\langle i j\rangle} \geq r_{\langle i j\rangle}^{\prime}=r_{i j}^{\prime}+1$, so that $r_{i j}>r_{i j}^{\prime}$.

Now, choose some $\left(k_{1}, l_{1}\right) \in \Delta$ with $\left(k_{1}, l_{1}\right) \not \leq \Delta^{\prime}$; and choose $\left(k_{0}, l_{0}\right)<\left(k_{1}, l_{1}\right)$ so that the rectangle $\left[k_{0}, k_{1}\right] \times\left[l_{0}, l_{1}\right]$ is as large as possible with $r_{i j}>r_{i j}^{\prime}$ for $(i, j) \in\left[k_{0}, k_{1}-1\right] \times$ $\left[l_{0}, l_{1}-1\right]$. (The above remarks show that $k_{0}<k_{1}, l_{0}<l_{1}$.)

Claim: There exists $\left(i_{0}, j_{0}\right) \in\left[k_{0}, k_{1}-1\right] \times\left[l_{0}, l_{1}-1\right]$ with $m_{i_{0} j_{0}}>0$. Otherwise, we would have $m_{i j}=0$ inside this rectangle. By the maximality, there exists $(i, j)$ in the rectangle with $r_{i, l_{0}-1}=r_{i, l_{0}-1}^{\prime}$ and $r_{k_{0}-1, j}=r_{k_{0}-1, j}^{\prime}$. Then

$$
r_{k_{0}-1, l_{0}-1}=r_{k_{0}-1, j}+r_{i, l_{0}-1}-r_{i j}<r_{k_{0}-1, j}^{\prime}+r_{i, l_{0}-1}^{\prime}-r_{i j}^{\prime} \leq r_{k_{0}-1, l_{0}-1}^{\prime} .
$$

This contradicts $r_{i j} \geq r_{i j}^{\prime}$, establishing the claim.

Let us say a rectangle $[i, k] \times[j, l]$ has property $(\mathrm{R})$ if:

$$
\begin{gathered}
m_{i j}, m_{k l}>0, \quad(k, l) \in \Delta, \quad(k, l) \not \leq \Delta^{\prime}, \\
r_{s t}>r_{i j}^{\prime} \quad \text { for }(s, t) \in[i, k-1] \times[j, l-1],
\end{gathered}
$$

Our argument above shows that we may choose $\left(i_{0}, j_{0}\right)$ so that $\left[i_{0}, k_{1}\right] \times\left[j_{0}, l_{1}\right]$ has property (R). Next choose points $\left(i_{1}, j_{1}\right),\left(i_{2}, j_{2}\right), \ldots,\left(i_{t}, j_{t}\right)$, as many as possible, such that: the 
rectangles $\left[i_{0}, i_{s}\right] \times\left[j_{0}, j_{s}\right]$ all have property $(\mathrm{R})$, and

$$
\begin{gathered}
i_{1}<i_{2}<\cdots<i_{t}, \quad j_{1}>j_{2}>\cdots>j_{t}, \\
{\left[i_{s}, i_{s+1}-1\right] \times\left[j_{s+1}, j_{s}-1\right] \cap \Delta=\emptyset} \\
{\left[i_{s}, i_{s+1}-1\right] \times\left[j_{s+1}, j_{s}-1\right] \cap \Delta^{\prime}=\emptyset}
\end{gathered}
$$

for all $s$. That is, $\left(i_{1}, j_{1}\right),\left(i_{2}, j_{2}\right), \ldots,\left(i_{t}, j_{t}\right)$ are consecutive elements of $\Delta$ (listed from northeast to southwest), not separated by intermediate elements of $\Delta^{\prime}$. By moving $\left(i_{0}, j_{0}\right)$ southeast if necessary, and keeping only the rectangles with $\left(i_{0}, j_{0}\right) \leq\left(i_{s}, j_{s}\right)$, we may assume, in addition to all the above, that $m_{i j}=0$ for all $(i, j) \neq\left(i_{0}, j_{0}\right)$ in the region

$$
S:=\bigcup_{s=1}^{t}\left[i_{0}, i_{s}-1\right] \times\left[j_{0}, j_{s}-1\right],
$$

the union of all our rectangles.

Now apply move (v) to the region $\left[i_{0}, i_{1}\right] \times\left[j_{0}, j_{t}\right]$ of $(M, \Delta)$, which contains all the $\left[i_{0}, i_{s}\right] \times\left[j_{0}, j_{s}\right]$ as subrectangles, and obtain $(M, \Delta) \underset{<}{(\mathrm{v})}(\tilde{M}, \tilde{\Delta})$. Then $\tilde{r}_{i j}=r_{i j}$ outside $S$, and $\tilde{r}_{i j}=r_{i j}-1 \geq r_{i j}^{\prime}$ inside $S$. Hence $\tilde{r}_{i j} \geq r_{i j}^{\prime}$ for all $(i, j)$.

Further, for $(i, j) \notin S$, we have $\delta_{i j}(\tilde{\Delta})=\delta_{i j}(\Delta)$, so clearly $\tilde{r}_{\langle i j\rangle}=r_{\langle i j\rangle} \geq r_{\langle i j\rangle}^{\prime}$.

Finally, consider $(i, j) \in S$. The definition of $\tilde{\Delta}$ and $S$ ensures that $\delta_{i j}(\tilde{\Delta})=0 \Rightarrow$ $\delta_{i j}\left(\Delta^{\prime}\right)=0$; if this were not so, we could enlarge the list $\left(i_{1}, j_{1}\right), \ldots,\left(i_{t}, j_{t}\right)$ while keeping $\left(i_{0}, j_{0}\right)$ fixed. Hence for $(i, j) \in S$, we have:

$$
\tilde{r}_{\langle i j\rangle}=\tilde{r}_{i j}+\delta_{i j}(\tilde{\Delta}) \geq r_{i j}^{\prime}+\delta_{i j}\left(\Delta^{\prime}\right)=r_{\langle i j\rangle}^{\prime} .
$$

That is, $\tilde{r}_{\langle i j\rangle} \geq r_{\langle i j\rangle}^{\prime}$ for all $(i, j)$, and $(\tilde{M}, \tilde{\Delta}) \stackrel{\text { rk }}{\leq}\left(M^{\prime}, \Delta^{\prime}\right)$.

This concludes the proof of Lemma 4 , and hence of the Rank and Move Theorems.

\section{Minimality}

In this section, we prove the Minimality Theorem for triple flags. In the case of full flags $\mathbf{b}=\mathbf{c}=\left(1^{n}\right)$ considered in the Introduction, this follows from the fact that each simple move corresponds to a codimension-one containment of orbits $F_{M, \Delta} \subset \bar{F}_{M^{\prime}, \Delta^{\prime}}$. However, this is not true in general. We give an alternative purely combinatorial argument.

By the Move Theorem, only the moves (i)-(v) are candidates for covers of our Bruhat order: we must show that each such move is indeed a minimal relation.

We will denote the Bruhat order on decorated matrices simply by $(M, \Delta) \leq\left(M^{\prime}, \Delta^{\prime}\right)$. We retain the notations $m_{i j}, m_{i j}^{\prime}, \tilde{m}_{i j}$ for the matrix entries of $M, M^{\prime}, \tilde{M}$; and $r_{i j}, r_{i j}^{\prime}, \tilde{r}_{i j}$ for the rank-numbers $r_{i j}(M), r_{i j}\left(M^{\prime}\right), r_{i j}(\tilde{M})$.

Given a decorated matrix $(M, \Delta)$, suppose we perform some simple move on the block $\left[i_{0}, i_{1}\right] \times\left[j_{0}, j_{1}\right]$, obtaining $\left(M^{\prime}, \Delta^{\prime}\right)$. We say a position $(i, j) \in[1, q] \times[1, r]$ is $M$-inactive 
with respect to the move $(M, \Delta)<\left(M^{\prime}, \Delta^{\prime}\right)$ if:

$$
r_{i j}=r_{i j}^{\prime}, r_{i-1, j}=r_{i-1, j}^{\prime}, r_{i, j-1}=r_{i, j-1}^{\prime}, r_{i-1, j-1}=r_{i-1, j-1}^{\prime}
$$

Otherwise $(i, j)$ is $M$-active. Similarly, $(i, j)$ is $\Delta$-inactive if:

$$
r_{\langle i-1, j-1\rangle}=r_{\langle i-1, j-1\rangle}^{\prime}, r_{\langle i-1, j\rangle}=r_{\langle i-1, j\rangle}^{\prime}, r_{\langle i, j-1\rangle}=r_{\langle i, j-1\rangle}^{\prime} ;
$$

and otherwise $(i, j)$ is $\Delta$-active. The following result implies that we may obtain $\left(M^{\prime}, \Delta^{\prime}\right)$ from $(M, \Delta)$ by changing the entries of $M$ only at the $M$-active positions, and the elements of $\Delta$ only at the $M$-active and $\Delta$-active positions, leaving $M$ and $\Delta$ unchanged at all inactive positions.

Lemma 8 If $(i, j)$ is $M$-inactive, then $m_{i j}=m_{i j}^{\prime}$. If $(i, j)$ is $M$-inactive and $\Delta$-inactive, then $(i, j) \in \Delta \Leftrightarrow(i, j) \in \Delta^{\prime}$.

Proof: For the first statement, $m_{i j}=r_{i j}-r_{i-1, j}-r_{i, j-1}+r_{i-1, j-1}$. For the second statement, note that $(i, j) \in \Delta$ exactly when $(i, j) \leq \Delta$ and $(i+1, j),(i, j+1) \not \leq \Delta$.

Now suppose we have a possible couterexample to the Minimality Theorem, a single move relation $(M, \Delta) \stackrel{\text { mv }}{\leftarrow}\left(M^{\prime}, \Delta^{\prime}\right)$, with an intervening element $(\tilde{M}, \tilde{\Delta})$ in the Bruhat order:

$$
(M, \Delta)<(\tilde{M}, \tilde{\Delta}) \leq\left(M^{\prime}, \Delta^{\prime}\right)
$$

We must show that $(\tilde{M}, \tilde{\Delta})=\left(M^{\prime}, \Delta^{\prime}\right)$.

Since rank numbers must decrease at an active position, we have: if $(i, j)$ is $M$-active for the move $(M, \Delta)<(\tilde{M}, \tilde{\Delta})$, then $(i, j)$ is $M$-active for the move $(M, \Delta)<\left(M^{\prime}, \Delta^{\prime}\right)$; and similarly for $\Delta$-active positions. That is, the move $(M, \Delta) \lessdot(\tilde{M}, \tilde{\Delta})$ may act only at the active positions of $(M, \Delta)<\left(M^{\prime}, \Delta^{\prime}\right)$.

We now need only inspect the active positions of each possible move $(M, \Delta) \check{\mathrm{mv}}^{\mathrm{v}}\left(M^{\prime}, \Delta^{\prime}\right)$, and verify that there is no other move $(M, \Delta) \stackrel{\text { mv }}{\leftarrow}(\tilde{M}, \tilde{\Delta})$ which can be performed on the given active positions and which satisfies $(\tilde{M}, \tilde{\Delta}) \stackrel{\text { rk }}{<}\left(M^{\prime}, \Delta^{\prime}\right)$.

(i) If $(M, \Delta) \underset{<}{(\text { i) }}\left(M^{\prime}, \Delta^{\prime}\right)$, then there are no $M$-active positions, so that $\tilde{M}=M$. Hence $\Delta<\tilde{\Delta}<\Delta^{\prime}$, but this is clearly impossible since $m_{i j}=0$ for all $(i, j)$ with $(i, j) \not \leq \Delta,(i, j) \leq \Delta^{\prime},(i, j) \neq\left(i_{1}, j_{1}\right)$.

(ii) The active positions form the block $\left[i_{0}, i_{1}\right] \times\left[j_{0}, j_{1}\right]$. If $\Delta$ is disjoint from this block, then only other moves of type (ii) are possible, but there are none such.

If $\left(i_{0}, j_{0}\right) \in \Delta, m_{i_{0} j_{0}}>1$, we must also consider type (i) applied to $\left\{i_{0}\right\} \times\left[j_{0}, j_{1}\right]$ or $\left[i_{0}, i_{1}\right] \times\left\{j_{0}\right\}$ or $\left[i_{1}, i_{2}\right] \times\left[j_{0}, j_{1}\right]$, each of which leads to $(M, \Delta) \stackrel{\text { (i) }}{<}(\tilde{M}, \tilde{\Delta}) \nless\left(M^{\prime}, \Delta^{\prime}\right)$, since $\tilde{r}_{\left\langle i_{0}-1, j_{0}\right\rangle}=r_{i_{0}-1, j_{0}}<r_{i_{0}-1, j_{0}}+1=r_{\left\langle i_{0}-1, j_{0}\right\rangle}^{\prime}$. Also possible is type (iii)(a) applied to $\left[i_{0}, i_{1}\right] \times\left[j_{0}, j_{1}\right]$, but then $(\tilde{M}, \tilde{\Delta}) \nless\left(M^{\prime}, \Delta^{\prime}\right)$, as above. We apply the transposed arguments for (iii)(b).

Similarly for the cases $\left(i_{0}, j_{1}\right) \in \Delta$ and $\left(i_{1}, j_{0}\right) \in \Delta$. 
(iii)(a) The $M$-active positions are the block $\left[i_{0}, i_{1}\right] \times\left[j_{0}, j_{1}\right]$, and the $\Delta$-active positions are those $(i, j) \in\left[i_{0}, i_{1}\right] \times\left[1, j_{1}\right]$ with $(i, j) \not \leq \Delta$. The only other possible moves are of type (i). If we apply move (i) to any block inside $\left[i_{0}, i_{1}\right] \times\left[1, j_{0}-1\right]$, then we must have $\Delta<\tilde{\Delta}<\Delta^{\prime}$, which is impossible. If we apply (i) to $\left[i_{0}, i_{1}\right] \times\left[j_{0}, j_{1}\right]$, then $(M, \Delta) \stackrel{(\mathrm{i})}{<}(\tilde{M}, \tilde{\Delta}) \nless\left(M^{\prime}, \Delta^{\prime}\right)$ as before. Transpose for (iii)(b).

(iv)(a) The $M$-active positions are $\bar{R}=\left[i_{0}, i_{1}\right] \times\left[j_{0}, j_{1}\right] \cup\left[i_{2}, i_{1}\right] \times\left[j_{2}, j_{1}\right]$, whereas the $\Delta$-active positions are $(i, j) \in\left[i_{2}, i_{1}\right] \times\left[j_{0}, j_{1}\right]$ with $(i, j) \not \leq \Delta$. The other possible moves are (iii) applied to $\left[i_{2}, i_{1}\right] \times\left[j_{0}, j_{2}\right]$ or to $\left[i_{2}, i_{0}\right] \times\left[j_{0}, j_{1}\right]$; or (i) applied to these same blocks. All of these give $(\tilde{M}, \tilde{\Delta}) \nless\left(M^{\prime}, \Delta^{\prime}\right)$.

(iv)(b) The active positions are $\left[i_{0}, i_{1}\right] \times\left[j_{0}, j_{1}\right]$. One possible move is (i) applied to $\left[i_{2}, i_{1}\right] \times$ $\left[j_{0}, j_{1}\right]$. In this case $\tilde{r}_{\left\langle i_{2}, j_{0}-1\right\rangle}=r_{\left\langle i_{2}, j_{0}-1\right\rangle}-1<r_{\left\langle i_{2}, j_{0}-1\right\rangle}=r_{\left\langle i_{2}, j_{0}-1\right\rangle}^{\prime}$, so $(\tilde{M}, \tilde{\Delta}) \not$ $\left(M^{\prime}, \Delta^{\prime}\right)$.

Another possibility is (iii)(a) applied to $\left[i_{2}, i_{1}\right] \times\left[j_{0}, j_{1}\right]$. Again $\tilde{r}_{\left\langle i_{0}-1, j_{0}\right\rangle}<r_{\left\langle i_{0}-1, j_{0}\right\rangle}^{\prime}$ and $(\tilde{M}, \tilde{\Delta}) \not \leq\left(M^{\prime}, \Delta^{\prime}\right)$. Similarly for (iii)(b) applied to $\left[i_{2}, i_{1}\right] \times\left[j_{0}, j_{1}\right]$.

(iv)(c) Transpose of (iv)(b).

(v). The active positions are: $\bigcup_{s=1}^{t}\left[i_{0}, i_{s}\right] \times\left[j_{0}, j_{s}\right]$. One possible move is (ii) applied to some block $\left[i_{0}, i_{s}\right] \times\left[j_{0}, j\right]$ for $j_{s+1} \leq j \leq j_{s}$. Then $\tilde{r}_{\left\langle i_{0} j_{0}\right\rangle}=r_{\left\langle i_{0} j_{0}\right\rangle}-1<r_{\left\langle i_{0} j_{0}\right\rangle}=$ $r_{\left\langle i_{0} j_{0}\right\rangle}^{\prime}$, so $(\tilde{M}, \tilde{\Delta}) \not \leq\left(M^{\prime}, \Delta^{\prime}\right)$. Similarly for (ii) applied to a block $\left[i_{0}, i\right] \times\left[j_{0}, j_{s}\right]$.

The only other possible move is (v) applied to some smaller block $\left[i_{0}, i_{l}\right] \times\left[j_{0}, j_{m}\right]$, where $[l, m] \subset[1, t]$ (strict inclusion). Then $\tilde{r}_{\left\langle i_{l-1} j_{m+1}\right\rangle}=r_{\left\langle i_{l-1} j_{m+1}\right\rangle}-1+1<r_{\left\langle i_{l-1} j_{m+1}\right\rangle}+1=$ $r_{\left\langle i_{l-1} j_{m+1}\right\rangle}^{\prime}$, because $\left(i_{0}, j_{0}\right)<\left(i_{l-1}, j_{m+1}\right)$. Hence $(\tilde{M}, \tilde{\Delta}) \not{\leq}\left(M^{\prime}, \Delta^{\prime}\right)$.

Minimality is thus proved.

\section{Acknowlegment}

This work grew out of a project with Jerzy Weyman and Andrei Zelevinsky. The author is indebted to them for suggesting the topic, as well as numerous helpful suggestions.

\section{Notes}

1. This fact was also noted by Brion [2]. More generally, our work [5] uses the theory of quiver representations to classify all products of partial flag varieties having finitely many orbits of $\mathrm{GL}_{n}$, and see also [6] for the case of the symplectic and orthogonal groups. Our case of two flags and a line is the simplest of these finite-orbit cases beyond the double flag varieties. Even for the other triple flag varieties of $\mathrm{GL}_{n}$, the combinatorial complexity of the degenerations seems to grow formidably.

2. That is, $F^{\prime}$ degenerates to $F$ if we can find a continuous family of configurations $\left(A(\tau), B_{\bullet}(\tau), C_{\bullet}(\tau)\right)$ indexed by a parameter $\tau \in \mathbb{C}$, such that the configurations for $\tau \neq 0$ are all of type $F^{\prime}$, but for $\tau=0$ we enter type $F$.

3. Note that these limits are guaranteed to exist by the properness of $\mathbb{P}^{n-1} \times \operatorname{Flag}(\mathbf{b}) \times \operatorname{Flag}(\mathbf{c})$. We do not need this general fact, however, since we explicitly identify the limit $\left(B_{\bullet}(0), C_{\bullet}(0)\right)$.

\section{References}

1. K. Bongartz, "On degenerations and extensions of finite dimensional modules," Advances in Mathematics 121 (1996), 245-287. 
2. M. Brion, "Groupe de Picard et nombres caractéristiques des variétés sphériques," Duke Mathematical Journal 58 (1989), 397-424.

3. P.H. Edelman, "The Bruhat order of the symmetric group is lexicographically shellable," Proceedings of the AMS 82 (1981), 355-358.

4. W. Fulton, Young Tableaux, Cambridge University Press, 1997.

5. P. Magyar, J. Weyman, and A. Zelevinsky, "Multiple flags of finite type," Advances in Mathematics 141 (1999), $97-118$.

6. P. Magyar, J. Weyman, and A. Zelevinsky, "Symplectic multiple flags of finite type," Journal of Algebra 230 (2000), 245-265.

7. C. Riedtmann, "Degenerations for representations of quivers with relations," Ann. Sci. Éc. Norm. Sup. 19(4) (1986), 275-301.

8. A. Skowronski and G. Zwara, "Degenerations in module varieties with finitely many orbits," Contemporary Mathematics 229 (1998), 343-356.

9. G. Zwara, "Degenerations of finite dimensional modules are given by extensions," Compositio Mathematica 121 (2000), 205-218. 